\title{
Erforschung potenzieller Entwicklungsverläufe diagnostischer Fähigkeiten angehender Chemielehrkräfte hinsichtlich Lernendenvorstellungen - Die Bedeutung individueller Vorstellungen über Lernendenvorstellungen
}

\author{
Julian Heeg $^{1}$ (D) $\cdot$ Robert Marten Bittorf ${ }^{1} \cdot$ Sascha Schanze $^{1}$ \\ Eingegangen: 27. Januar 2020 / Angenommen: 18. Januar 2021 / Online publiziert: 10. Februar 2021 \\ (c) Der/die Autor(en) 2021
}

\section{Zusammenfassung}

In den naturwissenschaftlichen Fächern ist die Diagnose von individuellen Lernendenvorstellungen aufgrund ihrer Bedeutung für Lehr-Lernprozesse ein zentrales Element der Lehrkräfteprofessionalisierung. In vielen Modellierungen zum Professionswissen von Lehrkräften finden sich daher Elemente zum Umgang mit Lernendenvorstellungen. Zusätzlich existieren für einzelne naturwissenschaftliche Fächer erste ausführliche Beschreibungen der inhaltlichen und methodischen Güte von diagnostischen Fähigkeiten. Gleichzeitig zeigen einige Studien, dass angehende Lehrkräfte verschiedenste Schwierigkeiten mit einer Durchführung einer formellen Diagnose von Lernendenvorstellungen haben. Wenige Erkenntnisse existieren bislang in Bezug auf die Entwicklung derartiger diagnostischer Fähigkeiten. Erste Studien deuten außerdem darauf hin, dass sich (angehende) Lehrkräfte häufiger einer eher hindernisorientierten Perspektive hinsichtlich Lernendenvorstellungen zuordnen lassen. Über das Ausmaß des Einflusses einer solchen Perspektive auf die Diagnose von Lernendenvorstellungen ist bislang ebenfalls wenig bekannt.

Ausgehend von diesen beiden Desideraten hat dieser Beitrag daher zwei primäre Ziele: Erstens sollen Entwicklungsprozesse angehender Chemielehrkräfte hinsichtlich der Diagnose von Lernendenvorstellungen erforscht werden. Zweitens steht die Ermittlung des Einflusses individueller Vorstellungen über Lernendenvorstellungen u. a. auf die Diagnose im Fokus. Hierfür wurden sieben Bachelorstudierende (gymnasiales Lehramt Chemie) in drei Gruppen über den Zeitraum eines Semesters bei der Bearbeitung von drei unterschiedlichen Fallbeispielen videografiert. Zusätzlich wurden die erstellten schriftlichen Diagnosen eingesammelt. Die qualitativ inhaltsanalytische Auswertung der Daten zeigt, dass die Studierenden sich lediglich partiell in ihren diagnostischen Fähigkeiten entwickeln. Als eine mögliche Erklärung hierfür werden die Vorstellungen der angehenden Lehrkräfte über Lernendenvorstellungen diskutiert. Ausblickend werden Implikationen für die universitäre Lehrkräftebildung abgeleitet.

Schlüsselwörter Diagnose · Lernendenvorstellungen · Fallbasiertes Lernen · Einsatz von Videovignetten · Lehrkräfteprofessionalisierung 


\title{
Investigating Possible Development Processes of Preservice Chemistry Teachers' Diagnostic Skills Regarding Learners' Conceptions-The Influence of Individual Conceptions About Learners' Conceptions
}

\begin{abstract}
The diagnosis of learners' individual conceptions is a central element of science teachers' professionalism. This is due to the influence learners' conceptions have on teaching-learning processes. Accordingly, most models for teachers' professional knowledge contains elements for dealing with learners' conceptions. In addition, initial descriptions can be found in specific scientific domains, detailing descriptions of the content-related and methodological quality of diagnostic abilities. At the same time, some studies show that pre-service teachers (PTSs) show various difficulties in performing a formal diagnosis. Regarding the development of these diagnostic abilities, however, little is known so far.

In addition, some studies indicate that (pre-service) teachers have a more obstacle-oriented perspective regarding learners' conceptions. However, there is currently a research gap regarding the extent of the influence of such a perspective on the diagnosis of learners' conceptions.

Building on these two desiderates, this article has two primary goals: Firstly, to investigate PSTs development of their diagnostic abilities. Secondly, to determine the influence of PSTs conceptions about learners' conceptions on their diagnoses. Data was gathered by videotaping pre-service chemistry teachers in small groups while working on three different cases as well as collecting their written artefacts. Data was analyzed by using qualitative content analysis. The results indicate that single PSTs improve their diagnostic performance. PSTs' conceptions about conceptions are discussed as one central influence factor for diagnosing learners' conceptions. Implications for research and teaching in science teacher education are outlined.
\end{abstract}

Keywords Diagnosis $\cdot$ Learners' conceptions $\cdot$ Cased-based learning $\cdot$ Video vignettes $\cdot$ Teachers professional development

\section{Einleitung}

Die Diagnose lehr-lernrelevanter Lernendenmerkmale bzw. deren Ausprägung ist ein zentrales Element der Lehrkräfteprofessionalisierung (u. a. Artelt und Gräsel 2009; von Aufschnaiter et al. 2015; Brunner et al. 2006; Praetorius et al. 2012). Wissen und Fähigkeiten rund um die Diagnose solcher Merkmale finden sich daher in zahlreichen Modellen zur Lehrerprofessionalisierung (bspw. Brunner et al. 2006; Oser 1997; Shulman 1987; Weinert et al. 1990). Häufig werden die diagnostischen Fähigkeiten (angehender) Lehrkräfte in einem pädagogisch orientierten Kontext thematisiert (z. B. Horstkemper 2006; Ingenkamp und Lissmann 2008; Schrader 2013). In den letzten Jahren sind jedoch vermehrt fachdidaktisch orientierte Arbeiten entstanden, die zum Ziel haben, die Diagnose fachdidaktischer Inhalte zu beschreiben, zu modellieren oder zu fördern (z. B. von Aufschnaiter et al. 2015, 2017; Bartel und Roth 2017; Beretz et al. 2017; Dannemann et al. 2018; Dübbelde 2013; Heinrichs 2015; Hößle et al. 2017; Meister et al. 2020; Rath 2017).

Einen zentralen Forschungsaspekt innerhalb der naturwissenschaftlichen Didaktiken stellen die individuellen Lernendenvorstellungen dar (z.B. Gropengießer und Marohn 2018; Taber 2019). Hierbei wird üblicherweise (mehr oder weniger explizit) postuliert, dass eine konsequente Berücksichtigung von Lernendenvorstellungen zu einem größeren Lernerfolg führt (u.a. Chen et al. 2020; Duit et al. 2013;
Sadler et al. 2013; Taber 2019). Entsprechend finden sich auch in einer Vielzahl von Modellen zum fachdidaktischen Wissen Elemente hierzu (Park und Oliver 2008; Schmelzing et al. 2013). Für eine Berücksichtigung der individuellen Vorstellungen ist ihre erfolgreiche Diagnose im Vorfeld eine zentrale Voraussetzung (von Aufschnaiter und Alonzo 2018; Kattmann et al. 1997; Kerr et al. 2006; Reinfried et al. 2009). Jedoch zeigt die Studienlage seit mehreren Jahrzehnten konstant auf, dass (angehende) Lehrkräfte verschiedene Schwierigkeiten mit der Diagnose von und dem Umgang mit Lernendenvorstellungen haben (z.B. Cox et al. 2016; Morrison und Lederman 2003; Nussbaum 1981). Ferner verfügen sie häufig über eher defizit- bzw. fehlerorientierte Vorstellungen hinsichtlich Lernendenvorstellungen (z. B. Gomez-Zwiep 2008; Larkin 2012; Mellado 1997) bzw. über eine lehrkraft- und inhaltszentrierte Perspektive (z.B. Cain 2012; Klinghammer et al. 2016; Markic und Eilks 2008), welche sich ebenfalls auf die Gestaltung von Lehr-Lernprozessen auswirken kann.

Eine entsprechende Förderung im Rahmen der fachdidaktischen Lehrkräftebildung ist daher empfehlenswert (von Aufschnaiter et al. 2015; Beretz et al. 2017) und durch die Vorgaben der Kultusministerkonferenz auch erforderlich (Kultusministerkonferenz 2017). In Bezug auf die diagnostischen Fähigkeiten von angehenden Lehrkräften hinsichtlich Lernendenvorstellungen existieren bislang nur wenige Modellierungen (Rath 2017). Für die deutsch- 
sprachige Chemiedidaktik existiert nach dem aktuellen Wissensstand der Autoren keine umfassende Beschreibung der diagnostischen Fähigkeiten angehender Lehrkräfte in Bezug auf Lernendenvorstellungen und des Einflusses von individuellen Vorstellungen auf diese Prozesse.

Daher wurde hierzu eine Seminarkonzeption entwickelt, mit Chemielehramtsstudierenden durchgeführt und unter den beiden oben aufgeführten Aspekten in einer explorativen Studie analysiert.

\section{Theoretischer Hintergrund}

Dieser Beitrag folgt einer konstruktivistischen Perspektive auf Lehren und Lernen (u.a. Gerstenmaier und Mandl 1995; Gropengießer 1999; Riemeier 2007; Widodo und Duit 2004, 2005). Aus dieser Perspektive lassen sich für das folgende Vorhaben drei zentrale Annahmen ableiten: (1) Lernen ist gleichzeitig ein individueller, selbstgesteuerter und sozialer Prozess, der auf den vorherigen individuellen Vorstellungen aufbaut. (2) Vorstellungen können nicht direkt weitergegeben werden - sie müssen stets aus Externalisierungen (Aussagen, Zeichnungen etc.) in einem interpretativen Prozess konstruiert werden. Als unmittelbare Konsequenz aus den beiden Annahmen ergibt sich, dass (3) die vorhandenen individuellen Lernendenvorstellungen diagnostiziert werden sollten, um sie gezielt für die Ausgestaltung eines möglichst effektiven Lehr-Lernprozesses berücksichtigen zu können.

Für den weiteren Verlauf wird außerdem angenommen, dass das Konstrukt Diagnose als mehrdimensional (z. B. Ingenkamp und Lissmann 2008) sowie als gegenstands- bzw. themenspezifisch zu betrachten ist (vgl. hierzu auch Dübbelde 2013; Hoppe et al. 2020; Rath 2017). Aus diesen Annahmen folgt u.a., dass weitere Faktoren (bspw. individuelle Vorstellungen über den Prozess der Diagnose bzw. den Gegenstand selbst) eine zentrale Rolle bei der Diagnose spielen. Daher wird im Folgenden zunächst geklärt, was in diesem Beitrag unter Lernendenvorstellungen als Ausgangspunkt für Lehr-Lernprozesse verstanden wird. Dies umfasst dabei sowohl Lernendenvorstellungen als Diagnosegegenstand (Zielgruppe Schülerinnen und Schüler) als auch die Rolle von Lernendenvorstellungen bei der Gestaltung von Lehr-Lernprozessen und bei der Diagnose selbst (Zielgruppe (angehende) Lehrkräfte). Anschließend werden ausgehend vom Gegenstand der Lernendenvorstellungen die Grundlagen der pädagogischen Diagnostik adaptiert und mit bereits existierenden Modellierungen zur Diagnose von Lernendenvorstellungen in Beziehung gesetzt. Dieser letzte Schritt ist - wie oben bereits erwähnt - notwendig, da bislang nur in wenigen Arbeiten Modellierungen zum Themenbereich Diagnose von Lernendenvorstellungen beschrieben werden (Hoppe et al. 2020; Rath 2017). Den Ab- schluss der theoretischen Einleitung bilden Möglichkeiten zur Förderung der diagnostischen Fähigkeiten mittels Videovignetten im Rahmen des fallbasierten Lernens.

\section{Lernendenvorstellungen als Ausgangspunkt für die Gestaltung von Lehr-Lernprozessen}

Unter Lernendenvorstellungen werden in diesem Beitrag alle individuellen gedanklichen Prozesse aufgefasst, über die die Lernenden zu einem spezifischen Kontext, Thema o. ä. verfügen können (z. B. Baalmann et al. 2004; Riemeier 2005). Dies umfasst alle Vorstellungen unabhängig davon, ob diese von den Individuen in einer Situation erstmalig ad hoc oder ob diese in vergleichbaren Situationen wiederholt konstruiert werden und somit als gefestigt angesehen werden können. Auch hinsichtlich der Personengruppen Schülerinnen und Schüler, Studierende etc. werden zunächst keine Unterscheidungen vorgenommen. Gleiches gilt für die Bewertung dieser Lernendenvorstellungen vor einem fachlichen bzw. fachdidaktischen Hintergrund. Um solche Lernendenvorstellungen zu kennzeichnen, die in einem spezifischen Kontext nicht mit den aktuellen wissenschaftlichen Vorstellungen übereinstimmen, werden diese im Folgenden wertfrei als alternative Lernendenvorstellungen bezeichnet (Wandersee et al. 1994). Im Rahmen einer konstruktivistischen Perspektive werden Lernendenvorstellungen als Lernausgangslage bzw. Lernmittel und nicht rein defizitär als Hindernisse betrachtet (Baalmann et al. 2004; Kattmann et al. 1997; Larkin 2012; Marohn 2008). Ein Lehr-Lernprozess, der ausschließlich gegen die vorhandenen (alternativen) Vorstellungen gestaltet ist, erscheint in diesem Licht wenig zielgerichtet (Özdemir und Clark 2007). Vielmehr sollten Vorstellungen im Hinblick auf ihre jeweilige Bedeutung im Lehr-Lernprozess hin eingeordnet werden. Dies wiederum kann die Beurteilung der Adäquatheit der Anwendungen einzelner Vorstellungen in einem spezifischen Kontext umfassen.

Hinsichtlich der Entstehung, der Komplexität sowie der Strukturierung (alternativer) Vorstellungen existieren verschiedene und teils widersprüchliche theoretische Ansätze (s. Özdemir und Clark 2007; Stark 2003). Ein Teil dieser Theorien geht davon aus, dass grundlegende Vorstellungen zumeist sensomotorischen bzw. sozialen Erfahrungen entstammen, die sich über Jahre hinweg in unterschiedlichen alltäglichen Kontexten bewährt haben (Niebert und Gropengießer 2015; Riemeier 2005). Eine unreflektierte Übertragung dieser grundlegenden, alltäglichen Vorstellungen auf fachliche Kontexte hat häufig die Entstehung alternativer Vorstellungen zur Folge (Chandrasegaran et al. 2008; Onwu und Randall 2006; Smith III et al. 1993). Bedingt durch die Viabilität der alltäglichen Vorstellungen ist eine von außen induzierte Veränderung zumeist stark gehemmt (Gropengießer und Marohn 2018). Innerhalb der naturwissenschaftli- 
chen Unterrichtsfächer treten alternative Vorstellungen häufig durch die Konfrontation mit nicht direkt wahrnehmbaren Bereichen zu Tage, wie bspw. einer submikroskopischen Ebene (z. B. Taber 2013). Analoges gilt für Vorstellungen über Lehr-Lernprozesse. Auch hier werden alltägliche Erfahrungen auf für uns nicht direkt erfahrbare Vorgänge unreflektiert übertragen. Hierzu zählen bspw. Vorstellungen, nach denen Wissen wie eine Ware unverändert weitergegeben werden kann (Jelemenska 2012; Leavy et al. 2007).

Lernendenvorstellungen im Chemieunterricht sind ein ausführlich erforschter Gegenstand (Duit 2009). So existieren zu unterschiedlichen Themenbereichen zahlreiche Studien, in denen alternative Lernendenvorstellungen beschrieben werden (bspw. Bain und Towns 2016; Heeg et al. 2020a; Grüß-Niehaus und Schanze 2011; Özmen 2004). Vergleichbares gilt für Vorstellungen (angehender) Lehrkräfte über die Gestaltung von Lehr-Lernprozessen. Auch hier existiert eine breite Studienlage (Al-Amoush et al. 2014; Brauer et al. 2008; Bryan 2012; Cheng et al. 2016; Hashweh 1996; Kember 1997; Leavy et al. 2007; Ling Wong et al. 2006; Löfström und Poom-Valickis 2013; Meyer 2004; Postareff et al. 2008). Bislang wurde jedoch kein Konsens hinsichtlich der Terminologie bzw. der Definition des zugrundeliegenden Konstruktes erreicht (Klinghammer et al. 2016; Pajares 1992). So werden als unterschiedliche Bezeichnungen bspw. Vorstellungen bzw. conceptions (z. B. Brauer et al. 2008), beliefs (z. B. Bryan 2012), orientations (z.B. Ekiz-Kiran und Boz 2020) oder auch Überzeugungen (z. B. Seidel et al. 2009) verwendet. Mit der jeweiligen Bezeichnung gehen teils unterschiedliche Definitionen einher (Klinghammer et al. 2016). Diese sind mitunter jedoch als inhaltlich deckungsgleich aufzufassen (Löfström und Poom-Valickis 2013). In Entsprechung zahlreicher - insbesondere deutschsprachiger ${ }^{1}$ - Studien wird auch hier der Terminus Vorstellungen bevorzugt (u. a. Brauer et al. 2008; Cheng et al. 2016; Fischler 2000a, 2000b; Jelemenska 2012; Kleickmann et al. 2010; Klinghammer et al. 2016; Koballa et al. 2000; Ling Wong et al. 2006; Markic und Eilks 2008; Mellado 1997; Meyer et al. 1999; Meyer 2004).

In den meisten Fällen werden in Bezug auf Vorstellungen über Lehr-Lernprozesse aufbauend auf Kember (1997) zwei übergeordnete Positionen verwendet, welche einerseits transmissive Vorstellungen umfassen, die stark auf die Lehrperson, den Inhalt und dessen Weitergabe durch die Lehrperson fokussieren und andererseits konstruktivistische Vorstellungen, in denen die Lernenden sowie deren Lernprozesse im Vordergrund stehen (Kember 1997; Markic und Eilks 2008; Postareff et al. 2008). Brauer et al. (2008) weisen allerdings darauf hin, dass diese beiden Vorstellungen

\footnotetext{
${ }^{1}$ Klinghammer et al. (2016) weisen in diesem Zusammenhang auf Schwierigkeiten in der Übersetzung des Wortes beliefs ins Deutsche hin.
}

nicht zwangsläufig als Gegensätze aufgefasst werden müssen. Vielmehr handelt es sich bei den Vorstellungen über Lehren und Lernen um ein mehrdimensionales Konstrukt (Brauer et al. 2008; Voss et al. 2013). So können (angehende) Lehrkräfte kontextspezifisch über verschiedene Vorstellungen aus diesem Kontinuum verfügen (Brauer et al. 2008; Koballa et al. 2000; Postareff et al. 2008a; Voss et al. 2013). Als zentrale Quelle für Vorstellungen über Lehren und Lernen gelten berufsbiographische Prozesse (Schlichter 2012). Insbesondere die eigene Schulzeit wird vielfach in diesem Zusammenhang hervorgehoben (Fischler 2000a; Kagan 1992; Koballa et al. 2000; Pajares 1992).

Die meisten angehenden Lehrkräfte verfügen zu Beginn ihres Studiums über eher transmissiv geprägte Vorstellungen (Cain 2012; Klinghammer et al. 2016; Markic und Eilks 2008) - wenngleich es hierzu auch gegenteilige Berichte gibt (Brauer et al. 2008). Transmissive Vorstellungen stehen in einem deutlichen Widerspruch zu dem, was die aktuelle (fachdidaktische) Lehr-Lernforschung als zielführend für einen möglichst lernwirksamen Unterricht ansieht (Duit et al. 2013; Fensham et al. 2013; Patry 2016). Es ist entsprechend empfehlenswert, im Rahmen der Lehrkräftebildung Lernangebote zu schaffen, mittels derer die Studierenden ihre Vorstellungen in Richtung einer konstruktivistischen Perspektive weiterentwickeln können (Al-Amoush et al. 2014). Dabei fungieren die bestehenden Studierendenvorstellungen als jeweilige Lernausgangslage (Ling Wong et al. 2006; Lohmann 2006; van Dijk und Kattmann 2010). Entsprechend der langjährigen Erfahrungen, die die Studierenden mit Lehr-Lernprozessen in ihrem Leben gemacht haben (bspw. Schulunterricht), sind ihre Vorstellungen stark gefestigt und daher resistent gegenüber Veränderungen (Kagan 1992). Auch wenn widersprüchliche Ergebnisse in der Literatur hinsichtlich des Einflusses formaler Bildungsprozesse z.B. im Rahmen von universitären Lehrveranstaltungen formuliert werden, so kann dennoch eine Veränderung von Vorstellungen über Lehren und Lernen innerhalb der Phasen der Lehrerbildung angenommen werden (Bryan 2012; Markic und Eilks 2013; Meyer et al. 1999; Schlichter 2012).

Vorstellungen über Lehr-Lernprozesse beziehen sich neben zahlreichen Aspekten u.a. auch auf Vorstellungen über Lernvoraussetzungen sowie über Lernprozesse (Putnam und Borko 1997). Noch spezifischer lassen sich verschiedene Verbindungen zwischen allgemeinen Vorstellungen über Lehren und Lernen sowie von Vorstellungen über Lernendenvorstellungen ausmachen. So berichten bspw. EkizKiran und Boz (2020), dass eine lehrpersonenzentrierte (transmissive) Perspektive auf Lehr-Lernprozesse die Analyse von Lernendenvorstellungen verhindern kann. Für den umgekehrten Fall zeigt bspw. Hashweh (1996) auf, dass konstruktivistisch geprägte Vorstellungen häufiger einhergehen mit Instruktionen, die auf eine Konzepterweiterung ausgerichtet sind. Vergleichbare Verbindungen finden sich 
Tab. 1 Vorstellungen über Lernendenvorstellungen

\begin{tabular}{lll}
\hline Positionen & Zugeordnete Vorstellungen & Quellen \\
\hline Lernendenvorstellungen (LV) sind & LV sind ausschließlich alternative LV & Barthmann et al. (2019), Davis \\
et al. (2006), Gomez-Zwiep & (2008), Ilyas und Saeed (2018), \\
& LV sind falsch & Larkin (2012), Mellado (1997), \\
& LV sind Wissenslücken (2004), Pine et al. (2001) \\
& LV müssen ersetzt werden & Meyer \\
& LV müssen korrigiert werden & Ohne Instruktion verfestigen sich LV \\
& Diagnose von LV nicht notwendig & Barthmann et al. (2019), Gomez- \\
Lernendenvorstellungen sind & LV können fachlich korrekt/inkorrekt sein & Zwiep (2008), Pine et al. (2001) \\
& LV müssen weiterentwickelt werden & \\
& An LV kann angeknüpft werden & \\
& Kenntnis um LV dient der Anpassung des fachlichen Niveaus & \\
& Diagnose von LV ist gewinnbringend &
\end{tabular}

auch an anderer Stelle (Brauer et al. 2008; Otero und Nathan 2008; Yang et al. 2014).

Eine genauere Betrachtung der Vorstellungen (angehender) Lehrkräfte über Lernendenvorstellungen sowie den Umgang mit diesen zeigt ein breites Spektrum. Analog zu Vorstellungen über Lehren und Lernen können hier zwei Positionen beschrieben werden (Tab. 1).

Die Datenlage zu Vorstellungen über Vorstellungen zeigt, dass die defizitären Positionen deutlich überwiegen (Duit et al. 2008; Gomez-Zwiep 2008; Larkin 2012; Mellado 1997). Gomez-Zwiep (2008) berichtet in ihrer Studie bspw., dass Grundschullehrkräfte Vorstellungen haben, nach denen alternative Lernendenvorstellungen insbesondere ein Problem jüngerer Jahrgänge sind; bei älteren Lernenden können diese Vorstellungen hingegen leichter korrigiert werden. Viele (angehende) Lehrkräfte verfügen über Vorstellungen, nach denen alltägliche Erfahrungen, aber auch der vorherige Unterricht mögliche Quellen für alternative Vorstellungen sind (Gomez-Zwiep 2008; Meyer 2004; Naah 2015).

\section{Die Diagnose von Lernendenvorstellungen}

Der Diagnose lehr-lernrelevanter Merkmale wird eine Schlüsselrolle in der Gestaltung und Durchführung eines effektiven Unterrichts zugeschrieben (Artelt und Gräsel 2009; von Aufschnaiter et al. 2015; Horstkemper 2006; Praetorius et al. 2012; Schrader 2013). Ausgehend von einem Angebot-Nutzungs-Verhältnis zwischen der Gestaltung von Lehr-Lernprozesse durch Lehrende und deren Nutzung durch Lernende (Helmke 2009), hilft die genaue Kenntnis der Lernendenvoraussetzungen bei der Gestaltung eines bestmöglichen Angebots (Kunter und Trautwein 2013). Somit besteht eine enge (theoretische) Verbindung zwischen der Diagnose und der Unterrichtsqualitätsdimension der kognitiven Aktivierung. Bislang existiert jedoch keine einheitliche Studienlage hinsichtlich eines statis- tischen Zusammenhangs zwischen Diagnose und Unterrichtsqualität (Karst et al. 2014). Die empirische Datenlage wird insgesamt als ausbaufähig eingeschätzt. So verbleibt es häufig bei theoretischen Annahmen bzw. Postulaten (Praetorius und Südkamp 2017). Vor dem Hintergrund der Komplexität des Unterrichts ist dies jedoch auch wenig verwunderlich. Betrachtet man die umfangreichen Wirkungsgefüge im Rahmen des Angebot-Nutzungs-Modells (Helmke 2009) wird schnell deutlich, dass der Weg von einer Diagnose hin zu einer erhöhten Lernleistung auf Seiten der Lernenden über eine Vielzahl von weiteren Schritten verläuft und ebenso vielen Einflussfaktoren unterliegt.

Bislang existiert noch kein allgemein anerkanntes Rahmenmodell zur Beschreibung diagnostischer Vorgänge, wenngleich in jüngster Zeit vielversprechende Varianten publiziert wurden (z. B. von Loibl et al. 2020). Auch für den Gegenstand der Lernendenvorstellungen existieren bislang kein allgemein anerkannten Rahmenmodelle zu deren Diagnose (Rath 2017). Daher werden im folgenden Abschnitt ausgehend vom Gegenstand der Lernendenvorstellungen unterschiedliche Facetten der Diagnose formuliert und bei Möglichkeit mit Erkenntnissen aus der pädagogischepsychologischen Forschung sowie mit ersten bestehenden Modellen zum Themenbereich Lernendenvorstellungen (Marohn und Rohrbach 2013; Rath 2017) in Beziehung gesetzt. Ferner werden im Zuge dieses Prozesse Gütekriterien formuliert.

Das übergeordnete Ziel bei der Diagnose von Lernendenvorstellungen besteht in der Bestimmung einer möglichst genauen (individuelle) Lernausgangslage für die Gestaltung weiterer Lehr-Lernprozesse (Baalmann et al. 2004; Kattmann et al. 1997; Riemeier 2005). Der Fokus liegt entsprechend auf einer potential- oder ressourcenfokussierten Förderdiagnostik und weniger auf einer Selektions- bzw. Auslesediagnostik; auch, wenn dies nicht immer trennscharf unterschieden werden kann (Reiners 2017). Im Bereich der pädagogischen Diagnostik finden sich vergleichbare, wenn- 
gleich gegenstandsübergreifende, Auffassungen (Artelt und Gräsel 2009; Ingenkamp und Lissmann 2008). So betonen bspw. Baumert und Kunter (2006) im Zusammenhang mit der Diagnose im Unterricht, dass das Vorwissen der Lernenden Grundlage der Gestaltung der Lernprozesse sein sollte.

Zur Erfüllung dieses Ziels müssen Daten unter der Einhaltung spezifischer Gütekriterien gesammelt, interpretiert und selektiert werden (Heitzmann et al. 2019). Hinsichtlich der Einhaltung von Gütekriterien im Rahmen einer Diagnose wird in der Regel zwischen einer formellen, semiformellen und informellen Diagnose je nach Grad der Anwendung wissenschaftlicher Methoden, der Sicherstellung der Güte der Urteile sowie den Grad an Reflexionen unterscheiden (Hascher 2008; Schrader 2013). Insbesondere die semioder auch informelle Diagnose stehen hierbei in der Kritik, da sie auf der Grundlage einer unsystematischen Erhebung bzw. Auswertung der Daten basiert und häufig durch individuelle Vorstellungen und Überzeugungen in Bezug auf die Gestaltung eines Lehr-Lernprozesses beeinflusst wird (Hascher 2008). Diese beiden Diagnoseformen nehmen einen nicht unerheblichen Anteil des alltäglichen Handelns ein. Sie sollten jedoch aufgrund der geschilderten Schwierigkeiten um Aspekte einer formellen Diagnose ergänzt werden (Schrader 2013).

In der fachdidaktischen Forschung herrscht bislang kein Konsens zur Strukturierung von Lernendenvorstellungen (z. B. Özdemir und Clark 2007). So enthalten verschiedene prominente Ansätze Modellierungen, in denen unterschiedlich komplexe Vorstellungskonstrukte beschrieben werden (Chi 2013; diSessa 2008; Gropengießer 1997; Vosniadou 2013); sie werden von einzelnen, unverbundenen Vorstellungen bis hin zu komplexen und miteinander verknüpften, theorieähnlichen Systemen in vielen Komplexitätsgraden beschrieben. Die Autorinnen und Autoren betonen hier übereinstimmend, dass je nach Komplexitätsgrad unterschiedliche Maßnahmen für den Lernprozess erforderlich sind bzw., dass sich nur bestimmte Komplexitätsgrade für Änderungen eignen. Für den Kontext dieser Studie ist bedeutsam, dass eine Diagnose von Lernendenvorstellungen außerhalb von wissenschaftlichen Untersuchungen primär einen eng begrenzten und somit handhabbaren Inhaltsbereich adressieren sollte. In vielen empirischen Studien hat sich die Nutzung solch eines eng begrenzten Komplexitätsgrades als zielführend herausgestellt - insbesondere im Hinblick auf anschließende Fördermaßnahmen (Baalmann et al. 2004; Reinfried et al. 2013; Riemeier 2010). Die explizite Erwähnung eines eng begrenzten Inhaltsbereiches findet sich in keiner der erwähnten Modellierungen (Marohn und Rohrbach 2013; Naah 2015; Rath 2017). Auch in Beschreibungen zu diagnostischen Prozessen wird dies nur implizit erwähnt (Heitzmann et al. 2019). Am ehesten kann dies als eine weitere Differenzierung des Urteilsbereiches aufgefasst werden (McElvany et al. 2009). Eine Lernaus- gangslage sollte außerdem holistisch bestimmt werden. Dies schließt sowohl die fachlich adäquaten wie auch die fachlich inadäquaten (alternativen) Lernendenvorstellungen ein (Baalmann et al. 2004; Kattmann et al. 1997; Riemeier 2005).

Vorstellungen werden als individuelle Konstrukte beschrieben (Baalmann et al. 2004). Allerdings lassen sich aufgrund vergleichbarer Erfahrungen auch vergleichbare Vorstellungen interindividuell beschreiben. Beispielsweise zeigen verschiedene Reviews, dass einige Vorstellungen in verschiedenen Ländern, bei unterschiedlichen Personengruppen und mittels unterschiedlicher Methoden erhoben, dennoch in vergleichbarer Form vorliegen (bspw. Bain und Towns 2016; Heeg et al. 2020a; Özmen 2004). Im Sinne eines ökonomischen Umgangs sollten daher diese häufigen Vorstellungen den Lehrkräften für eine Unterstützung ihrer Diagnose zugänglich gemacht werden.

Die Externalisierung von Vorstellungen kann durch unterschiedliche Modalitäten unterstützt werden. So eignen sich bspw. Zeichnungen eher zur Externalisierung statischer Aspekte, während textliche Varianten von Lernenden primär zur Beschreibung von prozessbezogenen Aspekten verwendet werden (Akaygun und Jones 2014). Die Analyse unterschiedlicher Artefakte von Lernenden steht im Einklang mit von Aufschnaiter et al. (2015). Auch Heitzmann et al. (2019) betonen, dass im Rahmen einer Diagnose latente Konstrukte aus Beobachtungen abgeleitet werden müssen. Rath (2017) sowie Marohn und Rohrbach (2013) führen ebenfalls auf, dass verschiedene Artefakte zur Konstruktion einer Vorstellung herangezogen werden sollen. Zentral hierbei ist allerdings, dass von Externalisierungen höchstens auf potenziell vorliegende Vorstellungen geschlossen werden kann. Ein direktes Gleichsetzen von bspw. Aussagen und Vorstellungen ist mit einer konstruktivistischen Perspektive auf Lehren und Lernen nicht vereinbar (vgl. hierzu auch Gropengießer 1997).

Diagnosen können unterschiedliche personenbezogene Ebenen, wie bspw. eine lernendenglobale sowie eine lernendenindividuelle Ebene adressieren (McElvany et al. 2009). Mit beiden Lernendenebenen werden verschiedene Diagnosesituationen assoziiert (Karst 2012; Karst et al. 2014). Auch die Vorstellungsforschung fokussiert beide Ebenen in Abhängigkeit der Zielsetzung. So finden sich auf der einen Seite eine Vielzahl von detaillierten und zumeist qualitativen Erhebungen einzelner Lernender. Auf der anderen Seite werden mit standardisierten Instrumenten größere Gruppen an Lernenden hinsichtlich ihrer Vorstellungen analysiert (Gurel et al. 2015). Für Lehrkräfte können je nach vorliegender Diagnosesituation beide Varianten gewinnbringend sein. Für diesen Beitrag stehen die individuellen Lernendenvorstellungen im Vordergrund. Auch Rath (2017) betont, dass eine personenspezifische Analyse ein wichtiges Differenzierungskriterium ist. 
von Aufschnaiter et al. (2015) unterscheiden in ihrer Überblicksarbeit vier verschiedene Möglichkeiten der Diagnose (Status-, Prozess-, Veränderungs- sowie Verlaufsdiagnose), von welchen für den hiesigen Kontext die Statusdiagnose hervorzuheben ist. In der pädagogischen Diagnostik wird eine Statusdiagnose häufig als ein Verfahren zur Bestimmung stabiler Merkmale oder Zustände vordergründig im Sinne einer Selektions- oder Auslesediagnostik beschreiben (Schrader 2014; Siemes 2008). Da viele (alternative) Lernendenvorstellungen jedoch nicht als kontextübergreifend stabil angesehen werden können (Taber 2019) und keine Grundlage für eine Selektion bieten sollten, ist diese Auffassung vor dem Hintergrund des Diagnosegegenstands der Lernendenvorstellungen zu adaptieren. Eine Statusdiagnose von Lernendenvorstellungen sollte entsprechend aufbauend auf einzelnen Externalisierungen durchgeführt werden, die in einem kurzen zeitlichen Rahmen entstanden sind. Inwiefern diese als stabil angesehen werden können, kann nicht valide beurteilt werden. Hierfür müssen im Sinne einer Prozess- oder Veränderungsdiagnose weitere Artefakte interpretiert werden.

Eine Diagnose lehr-lernrelevanter Merkmale bzw. deren Ausprägungen sollte stets zielgerichtet auf eine anschließende Fördermaßnahme erfolgen (von Aufschnaiter et al. 2015; Schrader 2013). Die Wirksamkeit der Diagnose zeigt sich daher erst in den darauf aufbauenden Maßnahmen zur Unterstützung der Lehr-Lernprozesse (Reiners 2017). Eine aufeinander bezogene Umsetzung von Diagnose und Förderung kann bspw. durch die Verwendung des Modells der Didaktischen Rekonstruktion geschehen (z. B. Kattmann et al. 1997). Da es sich bei der Anwendung des Modells der Didaktischen Rekonstruktion um einen iterativen und rekursiven Prozess handelt, kann die Diagnose von Lernendenvorstellungen in den beiden Untersuchungsaufgaben der Lernpotentialdiagnose sowie der Didaktischen Strukturierung (wiederholt) Verwendung finden (z. B. Kattmann et al. 1997). Innerhalb der jeweiligen Untersuchungsaufgaben erfolgt eine Diagnose mit verschiedenen Schwerpunkten: Im Rahmen der Lernpotentialdiagnose liegt der Schwerpunkt auf der Ermittlung einer Lernausgangslage, innerhalb der Didaktischen Strukturierung primär auf der Ermittlung der Weiterentwicklung der Lernendenvorstellungen (z. B. Riemeier 2010).

\section{Der Umgang (angehender) Lehrkräfte mit Lernendenvorstellungen}

Viele (angehende) Lehrkräfte wissen zwar prinzipiell um die Bedeutung von Lernendenvorstellungen, zeigen aber gleichzeitig häufig deutliche Wissenslücken (Gomez-Zwiep 2008; Ilyas und Saeed 2018; Yang et al. 2014). So sind sie bspw. selten in der Lage eine umfangreiche Definition zu formulieren oder zeigen Schwierigkeiten häufige the- menbezogene alternative Lernendenvorstellungen zu nennen (Barthmann 2018; Gomez-Zwiep 2008; Ilyas und Saeed 2018). In der Regel neigen sie dazu, die Bedeutsamkeit von spezifischen Lernendenvorstellungen zu unterschätzen bzw. ihre Lernenden zu überschätzen (Diakidoy und Iordanou 2003). Vergleichbares zeigt sich, wenn es um die Diagnostik im Unterrichtsgeschehen oder die Verwendung von konstruktivistischen Lehr-Lernmethoden geht (s. hierzu auch Uhren et al. 2013). Auch hier werden unterschiedliche wissensbezogene sowie methodische Schwierigkeiten beschrieben (z. B. Larkin 2012; Naah 2015). Morrison und Lederman (2003) konnten aufzeigen, dass selbst wenn Lehrkräfte um die Bedeutung von Lernendenvorstellungen wissen und angeben, diese zu berücksichtigen, sie dennoch keinerlei Diagnosen in ihrem Unterricht durchführen. Da (angehende) Lehrkräfte selten Lernendenvorstellungen gezielt diagnostizieren, ist bislang auch wenig darüber bekannt, wie sie dies eventuell tun, von welchen Faktoren es abhängt und inwiefern diese Prozesse gefördert werden können.

\section{Der förderliche Einsatz von Videovignetten im Rahmen des fallbasierten Lernens}

Die Diagnose lehr-lernrelevanter Aspekte ist eine anspruchsvolle und anforderungsreiche Tätigkeit (Hascher 2008). Diagnostische Fähigkeiten müssen entsprechend erst von angehenden Lehrkräften erworben werden (Hascher 2008; Meister et al. 2020). Heitzmann et al. (2019) betonen den Mehrwert von simulierten Lernumgebungen zur Förderung diagnostischer Fähigkeiten. Um dies umzusetzen, müssen die entsprechenden Gegenstände (z.B. Verstehensprozesse von Lernenden) jedoch zunächst erfahrbar und analysierbar gemacht werden. Hierfür eignet sich insbesondere der Einsatz von Videos bzw. Videovignetten (von Aufschnaiter und Rogge 2010; Dannemann et al. 2019).

Videos werden in der Lehrpersonenbildung bereits seit Jahrzehnten eingesetzt (van Es und Sherin 2008) und mit zahlreichen und oft affektiven Aspekten wie einer hohen Motivation, einem gesteigerten Interesse oder einer wahrgenommene Authentizität in Verbindung gebracht (Brovelli 2014; Krammer und Reusser 2005; Oser et al. 2009). Neben Szenarien zur Förderung allgemeindidaktischer Elemente (z.B. classroom management) finden sich in den Fachdidaktiken auch solche zur Erhebung bzw. Förderung fachdidaktischer Fähigkeiten (angehender) Lehrkräfte (von Aufschnaiter et al. 2017; von Aufschnaiter und Alonzo 2018; Bartel und Roth 2017; Huang et al. 2011; Johnson und Cotterman 2015; Lin 2005; Olson et al. 2016). Unabhängig davon liegt der Fokus des verwendeten Materials jedoch häufig auf der Betrachtung und Analyse des Verhaltens einzelner Lehrpersonen (Gaudin und Chaliès 2015; Janik et al. 
2013). Ergänzend zeigen angehende Lehrkräfte teils deutliche Schwierigkeiten, in komplexen videografierten Unterrichtssituationen relevante Aspekte wahrzunehmen und korrekt vor einem theoretischen Hintergrund zu interpretieren (z.B. Berliner 2001; Carter et al. 1988; Colestock und Sherin 2009; Erickson 2011; Heitzmann et al. 2019; Levin et al. 2009; Schäfer und Seidel 2015; Star und Strickland 2008). Für eine Analyse von komplexen Lernendenmerkmalen unter einer fachdidaktischen Perspektive erweist sich diese Art von Videos als eher ungeeignet (von Aufschnaiter und Rogge 2010; Wildgans-Lang et al. 2020). Es empfiehlt sich daher, die Komplexität der gezeigten Situationen einerseits zu reduzieren und andererseits auf einzelne Inhalte zu fokussieren (Olson et al. 2016; Wildgans-Lang et al. 2020). Dies kann bspw. dadurch gelingen, dass ausschließlich einzelne Lernende in den Fokus authentischer Aufnahmen rücken, verbunden mit dem Ziel, deren Verstehensprozesse erfahrbar und schlussendlich analysierbar zu machen (von Aufschnaiter und Rogge 2010; Dannemann et al. 2019). Die Nutzung authentischer Lernprozesse für diese Zwecke spiegelt dabei eine weitgehende Konsensmeinung wider (z.B. Brophy 2004; Dannemann et al. 2019; Sherin et al. 2009). So heben bspw. Sherin et al. (2009) hervor, dass Lehrkräfte inszenierte Videos schnell als solche identifizieren und in der Folge diese Art Videos nicht ernst nehmen würden.

Die Verbindung von Videos bzw. Videovignetten mit Kontextdokumenten, zusätzlichen Informationen und Instruktionen zu einem Fallbespiel kann die Bearbeitung zielführend unterstützen (Bartel und Roth 2017). Bei der Konstruktion eines Fallbeispiels werden die einzelnen Bestandteile in einem forschungs- bzw. literaturgeleiteten Verfahren miteinander kombiniert (Merseth 1994; Upmeier zu Belzen und Merkel 2014). Analog zur Gestaltung der Videos soll das Produkt dabei Eigenschaften wie Authentizität, Erfassbarkeit und Relevanz aufweisen (Atria et al. 2006; Campbell 1996; Jeffries und Maeder 2005). Dies ist stets vor dem Hintergrund des Einsatzziels sowie der dargestellten Inhalte individuell auszugestalten (von Aufschnaiter et al. 2017).

Seago (2004) betont, dass Videos lediglich ein Werkzeug sind. Erst der konkrete Umgang mit ihnen entscheidet über ein lernförderliches Potenzial. Es sollten demnach genauso viele Ressourcen in die Entwicklung der Begleitmaterialien (z.B. Aufgaben) investiert werden, wie in die Entwicklung der Videos selbst (Krammer et al. 2006). Entsprechend lassen sich verschiedenste Beispiele in Bezug auf den weiteren Umgang im Rahmen der universitären Lehrkräfte mit Videos bzw. Videovignetten finden (s. bspw. Blomberg et al. 2013). Wenngleich es noch ungeklärte Fragen bezüglich der idealen Strategie im Umgang mit Videos im Rahmen der universitären Lehrkräftebildung gibt (Blomberg et al. 2014), so lässt sich dennoch ein Konsens hinsichtlich zwei- er Aspekte feststellen, die einen lernförderlichen Umgang mit Videos unterstützen: (1) Strukturierung des Bearbeitungsprozesses: Die bloße Aufforderung, bestimmte Aspekte der Unterrichtspraxis näher zu betrachten, ist weniger hilfreich (Barnhart und van Es 2015). Vielmehr ist eine gezielte Fokussierung der Betrachtung auf spezifische Elemente innerhalb eines Videos durch passgenaue Arbeitsaufträge gefordert (Barnhart und van Es 2015; Llinares und Valls 2009; Santagata und Angelici 2010). Daneben kann auch die Einbettung in eine komplexe instruktionale Lernumgebung (z.B. ein Seminar) lernförderlich wirken (z.B. Kleinknecht und Gröschner 2016). (2) Gemeinsame Bearbeitungs- und/oder Diskussionsprozesse: Die gemeinsame Bearbeitung von Videos bzw. eine begleitende Diskussion über den Bearbeitungsprozess und dessen Ergebnisse können zu der Entwicklung einer gemeinsamen Sprache führen, die es (angehenden) Lehrkräften ermöglicht, ihre teilweise impliziten Vorstellungen zu reflektieren und somit einer Externalisierung zugänglich zu machen (Borko et al. 2008; Krammer und Reusser 2005; Lin 2005). Dies kann sich insgesamt förderlich auf den Lernprozess auswirken (vgl. Colestock und Sherin 2009; Zhang et al. 2011) insbesondere bei der Förderung diagnostischer Fähigkeiten (Heitzmann et al. 2019). Gleiches gilt für Reflexionsprozesse hinsichtlich der eigenen Vorstellungen über Lehren und Lernen bzw. über Lernendenvorstellungen (Dannemann et al. 2018; Ling Wong et al. 2006). Letztere können Studierende jedoch vor größere Schwierigkeiten stellen bzw. verbleiben häufiger ohne eine gezielte Unterstützung auf einem deskriptiven oder wertenden Niveau (Hübner et al. 2007; Schaefer et al. 2012). Als Unterstützungsmaßnahme können bspw. Prompts oder strukturierte Leitfragen eingesetzt werden (Hübner et al. 2007; Schaefer et al. 2012). Die Lernförderlichkeit des Einsatzes von Prompts und Reflexionsphasen insbesondere im Kontext diagnostischer Fähigkeiten wurde in einer aktuellen Meta-Analyse bestätigt (Chernikova et al. 2020). Ferner sollte es den Studierenden ermöglicht werden, dass sie die Videovignetten - insbesondere im Kontext der Diagnose - wiederholbar und ohne zusätzlichen Handlungsdruck bearbeiten können (Dannemann et al. 2019; Rieu et al. 2020).

\section{Leitende Fragestellungen}

Den Autoren ist keine Studie bekannt, die im Rahmen einer universitären Förderung Entwicklungsverläufe von angehenden Chemielehrkräften hinsichtlich ihrer diagnostischen Fähigkeiten in Bezug auf Lernendenvorstellungen sowie den Einfluss individueller Vorstellungen auf solche Entwicklungen untersucht. Zur Veränderung von Lernendenvorstellungen existieren zwar widersprüchliche Berichte, eine Förderung scheint aber durchaus möglich zu sein 
Abb. 1 Übersicht über Verlauf des Seminares, den Einsatz der Fallbeispiele sowie die Datenerhebung

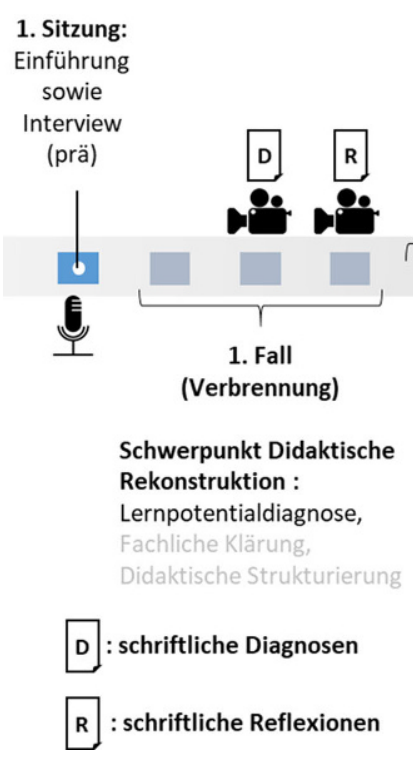

(Postareff et al. 2008b). Daher sollen im Rahmen dieser Studie die drei folgenden und explorativ ausgelegten Fragestellungen beantwortet werden:

F1 (Entwicklung der Vorstellungen) Über welche Vorstellungen über Lernendenvorstellungen sowie über deren Diagnose verfügen die Studierenden und inwiefern verändern sich diese Vorstellungen im Laufe der Studie?

F2 (Entwicklung der Diagnose) Wie verändert sich die Qualität der Diagnosen der Studierenden im Laufe der Studie?

F3 (bedeutungstragende Situationen) Welche bedeutungstragenden Situationen, die mit einem Lernerfolg assoziiert werden können, können im Verlauf der Intervention identifiziert werden?

\section{Design und Methoden}

\section{Entwicklung der Fallbeispiele und Konzeption der Lehrveranstaltung}

Für die Studie wurden in Anlehnung an die Vorgaben von Dannemann et al. (2019) drei unterschiedliche Videovignetten entwickelt, die als Fallbeispiele Verwendung fanden. Sie umfassen die Themenbereiche (1) chemische Reaktion am Beispiel einer Verbrennung von Kohle in einem geschlossenen System (z. B. Johannsmeyer et al. 2003; Petermann et al. 2010), (2) das chemische Gleichgewicht am Beispiel des Einflusses eines Katalysators auf ein solches System (Heeg et al. 2020a) sowie (3) die ionische Bindung am Beispiel des Lösens von Natriumchlorid in Wasser (z.B. Coll und Treagust 2003; Grüß-Niehaus und Schanze 2011; Taber

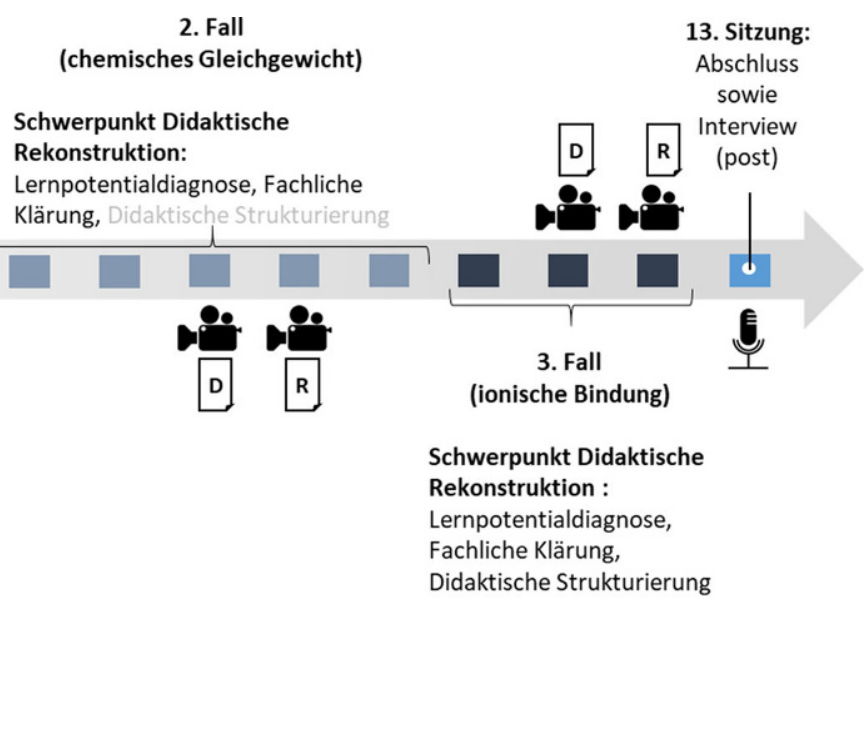

1997). Für die Entwicklung wurden jeweils zwei bis drei Schülerinnen und Schüler beim Austausch über einen entsprechenden fachlichen Inhalt videografiert. Grundlage für die Erhebungssituation war jeweils die Nutzung der PeerInteraction-Methode (Heeg et al. 2020b). Die aufgenommenen Videos wurden literaturgeleitet bearbeitet, sodass empirisch häufige alternative Lernendenvorstellungen erfahrbar bzw. analysierbar sind. Hierbei wurde einerseits darauf geachtet, dass nicht ausschließlich alternative Vorstellungen enthalten sind. Andererseits wurden die Vignetten so gestaltet, dass in der Regel mehrere Aussagen der Lernenden zur Konstruktion einer Vorstellung verwendet werden können. Bei der Auswahl an Aussagen wurde zusätzlich berücksichtigt, dass sie sich einander nicht widersprechen. Somit finden sich keine Anzeichen für eine Vorstellungsentwicklung in den jeweiligen Videovignetten, wodurch eine Statusdiagnose ermöglicht wird. Die Videovignetten wurden mit Kontextinformationen zu den Lernenden (z. B. Klassenstufe, letzte Chemienote) sowie Kontextdokumenten (Auszüge aus Schul- und Fachbüchern sowie aus den jeweiligen kerncurricularen Vorgaben, Transkript usw.) versehen.

Aufbauend auf diesen drei Fallbeispielen wurde eine Seminarkonzeption zur Förderung der diagnostischen Fähigkeiten angehender Chemielehrkräfte entwickelt (s. Heeg et al. im Druck). Dieses Seminar umfasste insgesamt 13 Sitzungstermine á $90 \mathrm{~min}$. Im Rahmen des Seminares wurde mit den Studierenden der Planungsrahmen der Didaktischen Rekonstruktion angewendet (Reinfried et al. 2009). Die jeweiligen Teilaufgaben der Didaktischen Rekonstruktion (Lernpotentialdiagnose, Fachliche Klärung sowie Didaktische Strukturierung) wurden hierbei schwerpunktartig im Rahmen der jeweiligen Fälle behandelt (s. Abb. 1). Eine schriftliche Diagnose der Lernendenvorstellungen wurde jedoch für jeden der drei Fälle gefordert. Zu jeder der 
Tab. 2 Verwendete Aufgabenstellungen sowie Leitfragen zur Unterstützung des Reflexionsprozesses

\begin{tabular}{|c|c|}
\hline Kategorie & Redigierte Aufgabenstellungen \\
\hline $\begin{array}{l}\text { Diagnose- } \\
\text { aufgaben }\end{array}$ & $\begin{array}{l}\text { „Stellen Sie sich vor, Sie sind Lehrkräfte an einem Gymnasium und müssen im nächsten Halbjahr das Thema chemische Re- } \\
\text { aktion am Beispiel Verbrennung und Massenerhalt unterrichten. Dafür wollen Sie u. a. auch den Boyle-Versuch als Demonstra- } \\
\text { tionsexperiment durchführen. Um einen Eindruck zu gewinnen, wie Ihre Lerngruppe darauf reagieren könnte, lassen Sie zwei } \\
\text { Schülerinnen ein Arbeitsblatt hierzu bearbeiten und filmen Sie dabei. } \\
\text { - Wie beurteilen Sie die Aussagen der beiden Schülerinnen in Bezug auf die Themen chemische Reaktion, Verbrennung und } \\
\text { Massenerhalt? } \\
\text { - Wie würden Sie die Lernenden unterstützen? Begründen Sie Ihre Antwort.“ }\end{array}$ \\
\hline Leitfragen & „Wie sind Sie bei der Bearbeitung der Aufgaben [...] vorgegangen?“ \\
\hline $\begin{array}{l}\text { Reflexions- } \\
\text { prozess }\end{array}$ & $\begin{array}{l}\text { „Vergleichen Sie Ihre Bearbeitung mit der Beispiellösung und klären Sie dabei die folgenden Fragen: } \\
\text { - Wo erkennen Sie Gemeinsamkeiten, wo Unterschiede? } \\
\text { - Wie bewerten Sie die Gemeinsamkeiten bzw. Unterschiede? } \\
\text { - Was würden Sie beim nächsten Mal genauso, was anders machen und warum?“ } \\
\text { „Welche selbst gesteckten Vorsätze aus der letzten Reflexion haben Sie dieses Mal berücksichtigt?““ }\end{array}$ \\
\hline
\end{tabular}

Teilaufgaben erhielten die Studierenden theoretische wie methodische Informationen (z. B. zu häufigen Lernendenvorstellungen oder zu Methoden zur Diagnose von Lernendenvorstellungen im Unterricht).

Alle Studierenden haben die gleichen Videovignetten in der gleichen Reihenfolge und mit den gleichen Aufgaben bearbeitet. Als Aufgabenstellung sollten die Studierenden aufbauend auf den theoretischen Grundlagen die Vorstellungen der Lernenden in der Vignette diagnostizieren und gleichzeitig angeben, wie sie die Lernenden unterstützen würden. Ein Teil der Aufgaben baut auf Rath (2017) auf. Nach jeder Bearbeitung erhielten die Studierenden u. a. eine Beispiellösung, anhand derer sie ihre Ergebnisse sowie ihre Vorgehensweise reflektieren sollten. Der Reflexionsprozess wurde dabei durch Leitfragen unterstützt (s. Tab. 2).

\section{Stichprobe und Datenerhebung}

Im Verlauf der im Jahr 2018 durchgeführten Studie wurden Daten von drei Studierendenkleingruppen über den Zeitraum eines Semesters erhoben (s. Tab. 3). Bei der hier vorliegenden Stichprobe handelt es sich um eine Gelegenheitsstichprobe (Döring und Bortz 2016). Die Teilnahme erfolgte freiwillig, die Studierenden haben sich selbst gruppiert.

Die Datenerhebung umfasst die bearbeiteten Aufgabenstellungen sowie schriftliche Reflexionsaufgaben (s. Abb. 1). Zusätzlich wurden die Austauschprozesse der Gruppen an insgesamt sechs Sitzungsterminen (jeweils zwei pro Fall) videografiert. Im Hinblick auf die Fragestellungen 1 und 3 wurden die Studierenden zusätzlich vor und nach dem Semester in Gruppen leitfadengestützt interviewt (Niebert und Gropengießer 2014). Die Interviews fanden dabei im Rahmen der ersten Sitzung bzw. im Anschluss an die letzte Sitzung statt und dauerten $28-35 \mathrm{~min}$ (prä) bzw. 14-25 min (post). Hierbei standen in dem vorab durchgeführten Interview primär ihre Vorstellungen über Lernendenvorstellungen und in dem abschließend durch- geführten Interview ihre Wahrnehmung hinsichtlich eines möglichen Lernerfolges im Fokus (s. Tab. 4).

\section{Datenauswertung}

Zur Beantwortung der Fragestellungen (s. Abschnitt Leitende Fragestellungen) wurden die Daten mittels einer inhaltlich strukturierenden qualitativen Inhaltsanalyse ausgewertet (Kuckartz 2018). Hierbei wurde einerseits eine vertiefende Fallanalyse und andererseits eine Kontrastierung verschiedener Fälle durchgeführt. Ferner wurde diese Art der qualitativen Inhaltsanalyse sowohl für die Auswertung der leitfadengestützten Interviews als auch für die Gruppendiskussionen eingesetzt. Nach Kuckartz (2018) ist der Schritt der Erstellung der Fallübersichten bzw. der vertiefenden Einzelfallinterpretation zentral für die Analyse der Daten. Ein wichtiges Merkmal dieses Schritts ist die Vergleichbarkeit zwischen den jeweiligen Fällen, u. a. verbunden mit dem Ziel einer möglichen Kontrastierung. Um die Vergleichbarkeit weiter zu erhöhen wurde dieser Analyseschritt bei der Auswertung der Daten im Hinblick auf die erste Fragestellung um eine Einzelstrukturierung (Gropengießer 2008) erweitert. Zur Beantwortung der zweiten Fragestellung wurde literaturgeleitet ein Kategoriensystem entwickelt und in Teilen induktiv ergänzt (s. Tab. 5). Heitzmann et al. (2019) weisen darauf hin, dass nicht die bloBe Anzahl an Diagnosetätigkeiten oder deren Reihenfolge die entscheidenden Gütekriterien sind, sondern vielmehr die Qualität der Einzeltätigkeiten selbst. Daher werden die jeweiligen Aspekte hier einzeln herangezogen und qualitativ inhaltsanalytisch betrachtet.

\section{Maßnahmen der Qualitätssicherung}

Im Bereich der qualitativen Forschung konnte bislang kein Konsens hinsichtlich der Formulierung einheitlicher Gütekriterien gefunden werden (Flick 2014). Für die hier angewendete Methode der qualitativen Inhaltsanalyse wer- 
den ebenfalls unterschiedliche Varianten vorgeschlagen (Kuckartz 2018; Mayring 2015). Aufbauend auf Empfehlungen aus der Literatur fokussieren qualitätssichernde Schritte vordergründig auf der Sicherstellung der Transparenz hinsichtlich der Datenerhebung und -auswertung sowie der Nachvollziehbarkeit der getätigten Interpretationen (Kuckartz 2018; Mayring 2015). Hierfür finden sich neben der Angabe der verwendeten Interviewfragen sowie Aufgabenstellungen und Reflexionsfragen auch die Angabe des Kategoriensystems zur Auswertung der Diagnoseergebnisse. Für die Auswertung wurden außerdem im Sinne einer Triangulation unterschiedliche Daten (schriftliche Bearbeitungen, Aussagen) herangezogen. Als letzter qualitätssichernder Schritt wurden die jeweiligen Interpretationen sowie die Einzelstrukturierung, welche vom Erstautoren durchgeführt wurden, aufbauend auf Flick (2014) kommunikativ mit weiteren Fachdidaktikerinnen und Fachdidaktikern $(N=3)$ validiert. Hierbei stand eine Konsensbildung im Vordergrund.

\section{Ergebnisse und Interpretation}

\section{Vorstellungen der Studierenden über Lernendenvorstellungen und Entwicklung ihrer diagnostischen Fähigkeiten}

In diesem Abschnitt werden Ergebnisse aus den Befragungen und den Dokumentationen der Fallbearbeitungen dargestellt. Dabei werden aufgrund der inhaltlichen Nähe Ergebnisse für die ersten beiden Fragestellungen gemeinsam aufgearbeitet und anschließend die dritte Fragestellung bearbeitet. Für die Beantwortung der ersten beiden Fragestellungen werden im Folgenden die Entwicklungsprozesse von zwei der drei Gruppen kontrastierend dargestellt. Als ausschlaggebende Kriterien wurden hierfür die anfänglichen Studierendenvorstellungen (prä Interview) über Lernendenvorstellungen sowie ihre Entwicklungsverläufe bzgl. der Diagnose herangezogen. Die Ergebnisse der Auswertung von Gruppe 2 werden abschließend vor dem Hintergrund der beiden Extremgruppen eingeordnet.

\section{Gruppe 1: Defizit- und fehlerorientierte Vorstellungen über Lernendenvorstellungen}

Die Studierenden dieser Gruppe zeigen im ersten Interview einen deutlich erkennbaren Zwiespalt zwischen ihren eigenen Vorstellungen über Lernendenvorstellungen sowie einer wahrgenommenen sozialen Erwünschtheit im Hinblick auf die zu verwendende Terminologie.

G1_S1_Interview_prä: Da hadere ich selbst immer noch so ein bisschen, so was jetzt, wie ich die falschen und die richtigen [Vorstellungen] abgrenze und was ich jetzt überhaupt sagen darf, was überhaupt fachlich korrekt ist.

Ihre eigenen Vorstellungen über Lernendenvorstellungen sind primär dadurch geprägt, dass sie diese als defizitär bzw. falsch ansehen (s. Tab. 6). Sie erkennen in ihnen eine gewisse Bedeutung für den Unterricht, fassen Lernendenvorstellungen allerdings als Lernhindernisse auf. Ferner erachten sie Wissenslücken als eine grundlegende Voraussetzung für die Entstehung von Lernendenvorstellungen. Diese Vorstellungen äußern die Studierenden kohärent über das gesamte Seminar hinweg. Anzeichen für eine Weiterentwicklungen ihrer Vorstellungen in Richtung von potential- bzw. ressourcenorientierten Vorstellungen finden sich kaum. In Bezug auf die Videovignetten werden die dort dargestellten alternativen Lernendenvorstellungen als primäres Merkmal hervorgehoben, die fachlich adäquaten Lernendenvorstellungen werden nicht erwähnt. Für die Studierenden dieser Gruppe umfasst die Diagnose von Lernendenvorstellungen vordergründig eine Suche nach Wissenslücken bzw. Verständnisschwierigkeiten. Dies steht im Einklang mit ihren weiteren Vorstellungen.

Im Rahmen ihrer ersten Diagnose handeln die Studierenden entsprechend ihrer Vorstellungen über Lernendenvorstellungen (s. Tab. 7). Für den Prozess selbst wählen sie einzelne Aussagen oder Aufgabenbearbeitungen der Lernenden aus und interpretieren diese, ohne den curricularen Rahmen zu kennen oder diesen zu recherchieren. Dies führt zu teilweise deutlichen Überinterpretationen bzw. der Anlegung eines nicht adäquaten Vergleichsrahmens.

Im Rahmen der Bearbeitung des zweiten Falls verändert sich ihr Diagnoseergebnis deutlich: Ihr Ergebnis weist eine hohe inhaltliche wie methodische Übereinstimmung mit

Tab. 3 Angaben zu den Studierenden

\begin{tabular}{|c|c|}
\hline Gruppe & Angaben \\
\hline \multirow[t]{3}{*}{ Gruppe 1} & Zwei männliche Studierende \\
\hline & $\begin{array}{l}\text { 4. Semester Bachelor (gymnasiales Lehramt Unter- } \\
\text { richtsfach Chemie) }\end{array}$ \\
\hline & $\begin{array}{l}\text { Drei abgeschlossene chemiedidaktischen Veranstaltun- } \\
\text { gen zu Beginn der Studie }\end{array}$ \\
\hline \multirow[t]{3}{*}{ Gruppe 2} & Zwei weibliche Studierende \\
\hline & $\begin{array}{l}\text { 4. Semester Bachelor (gymnasiales Lehramt Unter- } \\
\text { richtsfach Chemie) }\end{array}$ \\
\hline & $\begin{array}{l}\text { Drei abgeschlossene chemiedidaktischen Veranstaltun- } \\
\text { gen zu Beginn der Studie }\end{array}$ \\
\hline \multirow[t]{3}{*}{ Gruppe 3} & $\begin{array}{l}\text { Zwei weibliche Studierende, ein männlicher Studieren- } \\
\text { der }\end{array}$ \\
\hline & $\begin{array}{l}\text { 6. Semester Bachelor (gymnasiales Lehramt Unter- } \\
\text { richtsfach Chemie) }\end{array}$ \\
\hline & $\begin{array}{l}\text { Fünf abgeschlossene chemiedidaktischen Veranstaltun- } \\
\text { gen zu Beginn der Studie }\end{array}$ \\
\hline
\end{tabular}


Tab. 4 Auszüge aus dem Interviewleitfaden des ersten Interviews (vorher)

\begin{tabular}{|c|c|}
\hline Kategorie & Leitfragen sowie eventuelle Nachfragen \\
\hline \multirow[t]{2}{*}{$\begin{array}{l}\text { Verständnis/ } \\
\text { Definition }\end{array}$} & $\begin{array}{l}\text { „In der Chemiedidaktik wird sich viel mit den Vorstel- } \\
\text { lungen der Schülerinnen und Schüler beschäftigt. Be- } \\
\text { schreiben Sie bitte, was Sie unter diesen Vorstellungen } \\
\text { verstehen.“ }\end{array}$ \\
\hline & $\begin{array}{l}\text { „In der Literatur findet man verschiedene Bezeichnun- } \\
\text { gen für diese Vorstellungen }{ }^{\mathrm{a}}-\text { welche finden Sie am } \\
\text { besten und warum?“ }\end{array}$ \\
\hline \multirow[t]{4}{*}{$\begin{array}{l}\text { Herkunft/ } \\
\text { Quelle }\end{array}$} & $\begin{array}{l}\text { „Wie entstehen Ihrer Meinung nach diese Vorstellun- } \\
\text { gen?“ }\end{array}$ \\
\hline & Eventuelle Nachfragen: \\
\hline & „Existieren noch weitere Quellen?““ \\
\hline & $\begin{array}{l}\text { „Spielt der Unterricht hierbei eine Rolle? Wenn ja, } \\
\text { welche?““ }\end{array}$ \\
\hline \multirow[t]{2}{*}{ Umgang } & $\begin{array}{l}\text { „Inwiefern halten Sie persönlich Schülervorstellungen } \\
\text { für wichtig für Ihren späteren Unterricht?“ }\end{array}$ \\
\hline & $\begin{array}{l}\text { „Ich würde gerne mit Ihnen ein Gedankenspiel durch- } \\
\text { führen: Sie sind eine praktizierende Lehrkraft und in Ih- } \\
\text { rem Unterricht äußert ein Schüler/eine Schülerin etwas, } \\
\text { wohinter Sie eine fachlich nicht adäquate Vorstellung } \\
\text { vermuten. Wie würden Sie reagieren?“" }\end{array}$ \\
\hline
\end{tabular}

${ }^{a}$ Die Wortwahl wurde jeweils an die Terminologie der Studierenden angepasst. Wurde bspw. der Terminus Schülervorstellungen genutzt, so wurde dieser ebenfalls vom Interviewer durchgehend verwendet der zugrundeliegenden Beispiellösung auf und lässt eine Systematik erkennen (s. Tab. 8).

Die Bearbeitung des dritten Falls hingegen ähnelt wieder der des ersten Falls (s. Tab. 7). Im Zuge des Austauschprozesses sprechen die Studierenden mehrfach von fachlichen Aspekten, die bei den Lernenden „nicht präsent“ sind oder auch, dass die Lernenden „völlig falsche Vorstellung“ haben. Sie nutzen häufig ihr Wissen, dass alternative Vorstellungen durch Sprache bzw. Beobachtungen entstehen können.

\section{Fazit zu Gruppe 1}

$\mathrm{Zu}$ Beginn des Seminars verfügen die beiden Studierenden über eher fehler- bzw. defizitorientierte Vorstellungen über Lernendenvorstellungen (Fragestellung 1). Lernendenvorstellungen haben eine Bedeutung für sie, deren Berücksichtigung im Rahmen von Lehr-Lernprozessen wird ebenfalls betont. Ihr Fokus beschränkt sich jedoch auf fachlich inadäquate (alternative) Lernendenvorstellungen. Im Laufe des Seminares äußern die Studierenden an verschiedenen Zeitpunkten und im Zusammenhang mit unterschiedlichen Fällen vergleichbare Vorstellungen. Gleiches gilt für das abschließende Interview. Eine Weiterentwicklung findet entsprechend nicht statt.

Tab. 5 Kriteriensystem zur Beurteilung der Qualität (Güte) der Diagnosen

\begin{tabular}{|c|c|c|}
\hline \multirow[t]{2}{*}{ Kategorienbezeichnung } & Beschreibung & \multirow[t]{2}{*}{ Quellen } \\
\hline & $\begin{array}{l}\text { Im Rahmen dieser Kategorie wird analysiert, inwiefern die Studie- } \\
\text { renden in ihren Diagnosen ... }\end{array}$ & \\
\hline $\begin{array}{l}\text { I1: Fokus auf einzelne Vorstel- } \\
\text { lungen }\end{array}$ & $\begin{array}{l}\text {... auf einzelne thematisch eng begrenzte Inhaltsbereiche (i.d. R. ein- } \\
\text { zelne Konzepte/Aussagesätze) fokussieren }\end{array}$ & $\begin{array}{l}\text { In Anlehnung an die Formulie- } \\
\text { rung von Konzepten als Korngrö- } \\
\text { ße von Vorstellungen u. a. nach } \\
\text { Gropengießer (1997) }\end{array}$ \\
\hline I2: Fachliche Adäquatheit & $\begin{array}{l}\text {... sowohl fachlich adäquate wie fachlich inadäquate (alternative) } \\
\text { Vorstellungen der Lernenden angeben }\end{array}$ & $\begin{array}{l}\text { In Anlehnung an Kattmann et al. } \\
\text { (1997) }\end{array}$ \\
\hline I3: Fokus auf zentrale LV & $\begin{array}{l}\text {... auf zentrale (adäquate/inadäquate) Vorstellungen fokussieren bzw. } \\
\text { in einem zweiten Schritt selektieren. Hierfür wird die zugrundelie- } \\
\text { genden Beispiellösung als Vergleich herangezogen }\end{array}$ & $\begin{array}{l}\text { In Anlehnung an Hammann } \\
\text { und Asshoff (2017), Heeg et al. } \\
\text { (2020a) sowie Yang et al. (2014) }\end{array}$ \\
\hline I4: Inhaltliche Nachvollziehbar- & ... inhaltlich nachvollziehbare Diagnoseergebnisse (z. B. Vorstellun- & In Anlehnung an Rath (2017) \\
\hline
\end{tabular}

keit der Diagnoseergebnisse

M1: Trennung von Aussagen/ Aufgabenbearbeitungen und Vorstellungen (Konstruktion von Lernendenvorstellungen)

M2: Angabe von Belegen/ Bezügen

M3: Personenspezifische Diagnose

M4: Nutzung von verschiedenen Quellen (AB, Transkript etc.) gen) formulieren. Hierfür wird die zugrundeliegenden Beispiellösung als Vergleich herangezogen

... zwischen den Vorstellungen der Lernenden und ihren Aussagen bzw. Aufgabenbearbeitungen unterscheiden. Die Studierenden sollen Vorstellungen aufbauend auf den Aussagen bzw. Aufgabenbearbeitungen der Lernenden im Rahmen der Diagnose konstruieren

... ihre jeweiligen Diagnoseergebnisse korrekt durch die Angabe von Zeilennummern, einzelnen Aussagen, Verweisen auf Aufgabenbearbeitungen etc. belegen

... zwischen den einzelnen Lernenden in ihren Diagnosen unterscheiden. Sofern alle Lernenden über vergleichbare Vorstellungen verfügen, wird auch dies kenntlich gemacht

... alle zur Verfügung stehenden Materialien (Video, Transkript, Arbeitsblätter etc.) zur Diagnose nutzen
In Anlehnung an Gropengießer (1997), Heitzmann et al. (2019), Rath (2017)

Induktive Ergänzung

In Anlehnung an Karst (2012), Karst et al. (2014), McElvany et al. (2009), Rath (2017)

In Anlehnung an Akaygun und Jones (2014), von Aufschnaiter et al. (2015), Rath (2017) 


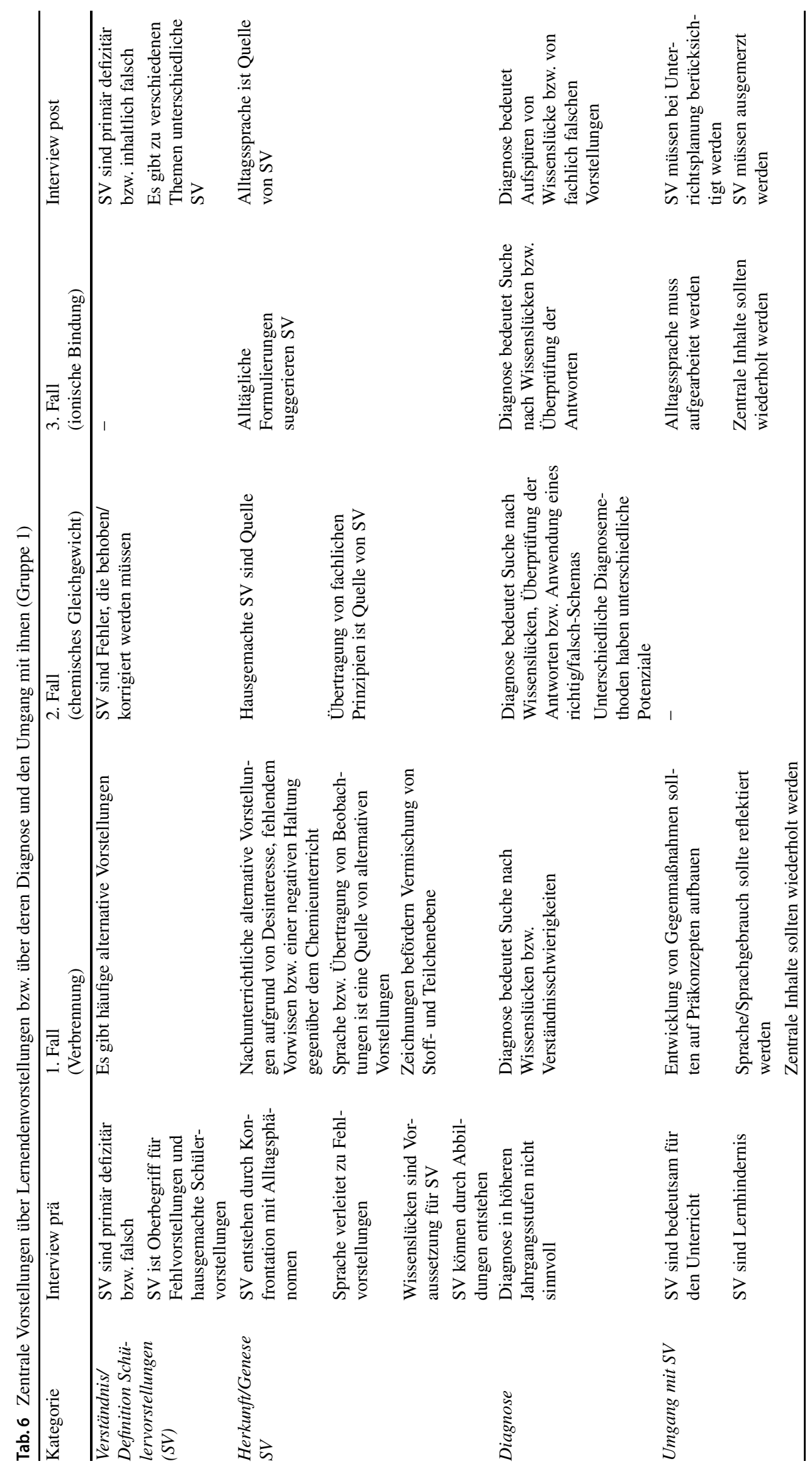


Tab. 7 Beschreibung inhaltlicher und methodischer Aspekte der Diagnose (Gruppe 1)

\begin{tabular}{|c|c|c|c|}
\hline & $\begin{array}{l}\text { 1. Fall } \\
\text { (Verbrennung) }\end{array}$ & $\begin{array}{l}\text { 2. Fall } \\
\text { (chemisches Gleichgewicht) }\end{array}$ & $\begin{array}{l}\text { 3. Fall } \\
\text { (ionische Bindung) }\end{array}$ \\
\hline \multirow[t]{4}{*}{$\begin{array}{l}\text { Inhaltliche } \\
\text { Aspekte }\end{array}$} & $\begin{array}{l}\text { Primär defizit- bzw. fehlerorientierte } \\
\text { Analyse von themenübergreifenden } \\
\text { Aspekten (I1 \& I2) }\end{array}$ & $\begin{array}{l}\text { Analyse von einzelnen thematisch eng } \\
\text { begrenzten fachlichen Vorstellungen } \\
\text { (I1) }\end{array}$ & $\begin{array}{l}\text { Primär defizit- bzw. fehlerorientierte } \\
\text { Analyse von themen-übergreifenden } \\
\text { Aspekten (I1 \& I2) }\end{array}$ \\
\hline & $\begin{array}{l}\text { Überinterpretation von einzelnen Aus- } \\
\text { sagen (I1) }\end{array}$ & $\begin{array}{l}\text { Gleichberechtigte Analyse von fachlich } \\
\text { adäquaten wie inadäquaten Lernenden- } \\
\text { vorstellungen (I2) }\end{array}$ & $\begin{array}{l}\text { Überinterpretation von einzelnen Aus- } \\
\text { sagen (I1) }\end{array}$ \\
\hline & $\begin{array}{l}\text { Geringe Übereinstimmung mit der } \\
\text { Beispiellösung (I4) }\end{array}$ & $\begin{array}{l}\text { Angabe von vergleichsweise wenigen } \\
\text { und nicht zentralen Vorstellungen (I3) }\end{array}$ & $\begin{array}{l}\text { Geringe Übereinstimmung mit der } \\
\text { Beispiellösung (I4) }\end{array}$ \\
\hline & & $\begin{array}{l}\text { Hohe inhaltliche Übereinstimmung mit } \\
\text { der Beispiellösung bei den angegebe- } \\
\text { nen Vorstellungen (I4) }\end{array}$ & \\
\hline \multirow[t]{3}{*}{$\begin{array}{l}\text { Methodische } \\
\text { Aspekte }\end{array}$} & $\begin{array}{l}\text { Keine systematische Konstruktion von } \\
\text { (alternativen) Vorstellungen (M1) }\end{array}$ & $\begin{array}{l}\text { Systematische Konstruktion von Vor- } \\
\text { stellungen (M1) }\end{array}$ & $\begin{array}{l}\text { Keine systematische Konstruktion von } \\
\text { (alternativen) Vorstellungen (M1) }\end{array}$ \\
\hline & $\begin{array}{l}\text { Keine hinreichende Angabe von Bele- } \\
\text { gen für die Diagnoseergebnisse (M2) }\end{array}$ & $\begin{array}{l}\text { Keine hinreichende Angabe von Bele- } \\
\text { gen/Quellen für die Diagnoseergebnis- } \\
\text { se (M2) }\end{array}$ & $\begin{array}{l}\text { Keine hinreichende Angabe von Bele- } \\
\text { gen für die Diagnoseergebnisse (M2) }\end{array}$ \\
\hline & $\begin{array}{l}\text { Keine systematische Trennung zwi- } \\
\text { schen den Lernenden (M3) }\end{array}$ & $\begin{array}{l}\text { Systematische Trennung zwischen den } \\
\text { Lernenden (M3) }\end{array}$ & $\begin{array}{l}\text { Keine systematische Trennung zwi- } \\
\text { schen den Lernenden (M3) }\end{array}$ \\
\hline
\end{tabular}

Tab. 8 Gegenüberstellung Studierendenbearbeitung zum zweiten Fall (chemisches Gleichgewicht) und Beispiellösung (Gruppe 1)

\begin{tabular}{|c|c|}
\hline Auszug aus der Aufgabenbearbeitung der Studierenden & Auszug aus der Beispiellösung \\
\hline \multicolumn{2}{|l|}{$\overline{A:}$} \\
\hline Stoffmengenänderung geht mit Gleichgewichtsverschiebung einher & Katalysatorzugabe stört/verschiebt Gleichgewicht ${ }^{\mathrm{a}}$ \\
\hline $\begin{array}{l}\text { GGW entsteht sobald die Konzentrationenänderungen [sic] der Edukte } \\
\text { und Produkte gleich ist }\end{array}$ & $\begin{array}{l}\text { Gleiche Konzentrationsänderung von Edukten und Produkten als } \\
\text { Voraussetzung für Gleichgewicht }\end{array}$ \\
\hline Katalysatoren binden und spalten & $\begin{array}{l}\text { Katalysator reagiert ausschließlich mit Edukt }{ }^{\text {a }} \\
\text { Katalysator spaltet sich von Edukt ab }\end{array}$ \\
\hline \multicolumn{2}{|l|}{$B:$} \\
\hline Katalysatoren begünstigen nur bei endothermen Reaktionen & Katalysator bevorzugt/begünstigt endotherme Reaktion ${ }^{\mathrm{a}}$ \\
\hline Katalysator begünstigt entweder Hin-, oder Rückreaktion & Katalysator begünstigt Hin- und Rückreaktion ${ }^{a}$ \\
\hline Stoffmenge verändert durch Katalysatorzugabe & Katalysatorzugabe verschiebt Gleichgewicht in Richtung Edukte ${ }^{a}$ \\
\hline GGW bedeutet nicht, dass die Konzentrationen gleich sein müssen & $\begin{array}{l}\text { Im Gleichgewicht liegen Edukte und Produkte nicht immer in glei- } \\
\text { chen Konzentrationen } \text { vor }^{\mathrm{a}}\end{array}$ \\
\hline Ein Katalysator hat verschiedene Gestalten & Ein Katalysator kann nicht gleichzeitig verbinden und spalten ${ }^{\mathrm{a}}$ \\
\hline
\end{tabular}

an der Beispiellösung als zentrale Vorstellung gekennzeichnet

Die jeweiligen Diagnoseprozesse bewegen sich primär im Spektrum einer informellen Diagnose (Fragestellung 2). Dies gilt insbesondere für den ersten und dritten Fall. Im Rahmen des zweiten Falls zeigen sich Veränderungen auf inhaltlicher und methodischer Ebene. Aufgrund der Übereinstimmung mit der Beispiellösung kann dies als teilweiser Anstieg einer Qualität der Diagnose im Sinne hier verwendeten Kategorien interpretiert werden. Jedoch führen die Studierenden auch hier keine hinreichenden Belege an oder reflektieren ihr Diagnoseergebnis. Ihre Vorgehensweise ist kongruent mit ihren defizit- bzw. fehlerorientierten Vorstellungen über Lernendenvorstellungen und deren Diagnose.

\section{Gruppe 3: Ressourcen- bzw. potentialorientierte Vorstellungen über Lernendenvorstellungen}

Die Studierenden erläutern im ersten Gruppeninterview anhand eines Beispiels, dass Lernendenvorstellungen für sie zwar wissenschaftlich nicht richtig sind, dass allerdings auf diesen Vorstellungen aufgebaut werden kann, sodass die Lernenden schlussendlich wissenschaftlich adäquate Vorstellungen bilden können (s. Tab. 9). Im ersten Interview geben die Studierenden außerdem an, dass das Einbeziehen von Lernendenvorstellungen für sie eine Bereicherung der Unterrichtsgestaltung darstellt. Die Rolle der Lehrkraft sehen sie zu Beginn darin, dass diese die Lernenden zur Bildung fachlich adäquater Vorstellungen anregen soll, indem eine passende Lernumgebung gestaltet wird. 


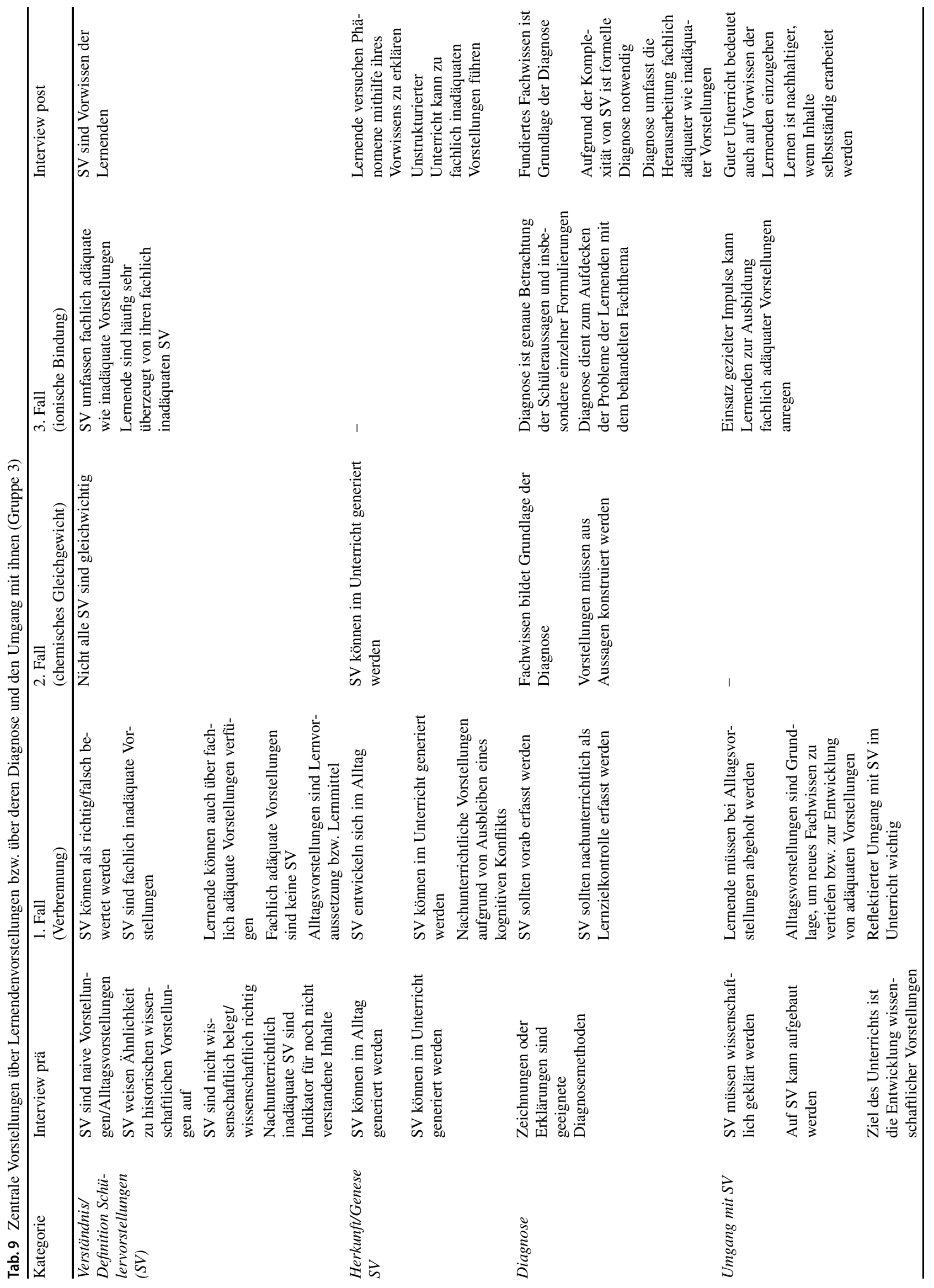


G3_S2_Interview_prä: Ich finde die Lernumgebung sollte mehr zeigen, dass vielleicht die eigenen Vorstellungen nicht wissenschaftlich sind und dass vielleicht die, dass sie, ja dass die mehr so auf die Vorstellungen geführt werden, die sie am Ende dann laut KC [Kerncurriculum] besitzen sollten.

Auffällig ist, dass die Studierenden im Rahmen der ersten Bearbeitung unter Lernendenvorstellungen ausschließlich Alltagsvorstellungen bzw. fachlich inadäquate (alternative) Vorstellungen verstehen, im Zuge des dritten Falls allerdings äußern sie, dass Lernendenvorstellungen (wie in der Lehrveranstaltung thematisiert) fachlich adäquate wie inadäquate Vorstellungen umfassen. Im abschließenden Interview zeigen die Studierenden eine differenzierte Auffassung der Diagnose von Lernendenvorstellungen im Unterricht. So beschreiben sie die Durchführung einer formellen Diagnose zwar als zeitintensiv und inhaltlich wie methodisch komplex, erkennen allerdings gleichzeitig ihre Notwendigkeit aufgrund der Komplexität der Lernendenvorstellungen.

Im Zuge der Bearbeitung des ersten Falls zeigen die Studierenden bereits deutlich erkennbare Ansätze für einen detaillierten und teilweise potentialorientierten Umgang mit den Vorstellungen der Lernenden in der Vignette.

G3_S1_1.Fall: Und die andere Schülerin hatte halt die Vorstellung ,Okay, das kann da ja nicht raus. Wir betrachten hier ein abgeschlossenes Reaktionssystem. Da kann nichts entweichen. Es kann nichts dazukommen. Also muss die Masse gleichbleiben. (...)“ Aber sie hatte eben das Problem, dass sie gedacht hat, dass sich nur der Aggregatzustand ändert, was man an ihrer Reaktionsgleichung sehen kann, dass die Edukte und die Produkte genau gleich sind.

In der nachfolgenden Reflexion der Fallbearbeitung betonen die Studierenden außerdem, dass sie die Diagnose von Schülervorstellungen und das Eingehen auf die Vorstellung für ein grundlegendes Element des Chemieunterrichts halten. Ohne diese Aspekte ist aus der Perspektive der Studierenden heraus ein Entwickeln fachlich adäquater Vorstellungen nicht möglich. Dieser ressourcen- bzw. potentialorientierte und letztlich auch wertschätzende Umgang mit den Vorstellungen der Lernenden zeigt sich ebenfalls bei der Bearbeitung der weiteren Fälle. Die Studierenden versuchen Gründe für das Vorhandensein der alternativen Lernendenvorstellungen abzuleiten und sich deren Entstehung somit zu erklären. Dabei wird deutlich, dass sie nicht nur eine Lernendenperspektive einnehmen können, sondern auch ihr eigenes Fachwissen kritisch hinterfragen (s. Tab. 10 und 11).

Das Diagnoseergebnis des dritten Falls gleicht dem des zweiten Falls. Auch hier zeigen die Studierenden eine Anwendung einer formellen Diagnose auf inhaltlicher wie methodischer Ebene (vgl. Tab. 11).

\section{Fazit zu Gruppe 3}

Die Studierenden der Gruppe 3 verfügen bereits im ersten Interview vor Beginn des Seminares über eher ressourcenbzw. potentialorientierte Vorstellungen über Lernendenvorstellungen (Fragestellung 1). Diese bauen sie im Laufe des Seminares partiell aus, sodass sie zum Ende hin ebenfalls über fachdidaktisch erwünschte Vorstellungen bspw. zur Diagnose verfügen (s. Tab. 9). Die Studierenden erkennen in der ihnen dargebotenen Vorgehensweise einen deutlichen Mehrwert für sich und nutzen diese auch (Fragestellung 2). $\mathrm{Ab}$ dem zweiten Fall fertigen sie konsequent formelle Diagnosen an. Dies zeigt sich insbesondere auch bei der Bearbeitung des dritten Falls. Die Studierenden nutzen für die Einordnung der Lernendenvorstellungen sowohl fachchemische Bücher als auch die curricularen Vorgaben zur Bestimmung eines Referenzrahmens.

G3_S2_3.Fall: [Ich] habe die Schülervorstellungen einmal aufgelistet, unterteilt in jeden Schüler und

Tab. 10 Auszüge aus den Diagnoseergebnissen der Gruppe 3

\begin{tabular}{|c|c|c|c|c|}
\hline \multirow[t]{2}{*}{$\begin{array}{l}\text { Auszug aus den Studierendenergebnissen des } \\
\text { ersten Falls (Verbrennung) }\end{array}$} & \multicolumn{2}{|c|}{$\begin{array}{l}\text { Auszug aus den Studierendenergebnissen } \\
\text { des zweiten Falls (chemisches Gleichge- } \\
\text { wicht) }\end{array}$} & \multicolumn{2}{|c|}{$\begin{array}{l}\text { Auszug aus den Studierendenergebnissen } \\
\text { des dritten Falls (ionische Bindung) }\end{array}$} \\
\hline & „Vorstellung Katalyse & Zeile & „Schülervorstellung & Beleg \\
\hline \multirow{4}{*}{$\begin{array}{l}\text { „Linke Schülerin: Aussage: ,Gewicht verringert } \\
\text { sich. 'Die Schülerin begründet ihre Aussage } \\
\text { indem sie anführt, dass Gase kein Gewicht haben } \\
\text { und folglich sich das Gesamtgewicht verringern } \\
\text { müsste. Diese Vorstellung lässt sich als } \\
\text { Schülervorstellung/Alltagsvorstellung } \\
\text { interpretieren. Des Weiteren lässt sich daraus } \\
\text { schließen, dass sie keine adäquate Vorstellung } \\
\text { zum Thema Massenerhaltung besitzt.“ }\end{array}$} & $\begin{array}{l}\text { Der Katalysator bevorzugt } \\
\text { nur eine Reaktionsrichtung }\end{array}$ & $1-2$ & $\begin{array}{l}\text { Lösevorgang ein aktiver } \\
\text { Prozess. Der Feststoff } \\
\text { mischt sich dazwischen }\end{array}$ & 3,10 \\
\hline & $\begin{array}{l}\text { Der Katalysator verschiebt } \\
\text { die Gleichgewichtslage }\end{array}$ & $3-4$ & $\begin{array}{l}\text { Das Wasser löst das Koch- } \\
\text { salz auf }\end{array}$ & 14 \\
\hline & $\begin{array}{l}\text { Der Katalysator geht } \\
\text { unverändert aus der } \\
\text { Reaktion hervor }\end{array}$ & $78^{\prime \prime}$ & $\begin{array}{l}\text { Bei festen Stoffen besitzen } \\
\text { die Teilchen einen geringen } \\
\text { Abstand zueinander }\end{array}$ & $44-45$ \\
\hline & & & $\begin{array}{l}\text { Bei Lösevorgang werden Io- } \\
\text { nen aus dem Gitter getrennt }\end{array}$ & $56^{\prime \prime}$ \\
\hline
\end{tabular}


Tab. 11 Beschreibung inhaltlicher und methodischer Aspekte des Diagnoseergebnisses (Gruppe 3)

\begin{tabular}{llll}
\hline & 1. Fall & 2. Fall & 3. Fall \\
(Verbrennung) & (chemische Bindung)
\end{tabular}

dann habe ich [die] halt mit dem Binnewies [Fachbuch für Allgemeine und Anorganische Chemie], teilweise mit dem Kerncurriculum [verglichen].

\section{Einordnung von Gruppe 2}

Die beiden Studierenden aus der Gruppe 2 verfügen über verschiedene Vorstellungen über Lernendenvorstellungen und deren Diagnose (z. B. SV sind nicht richtig oder falsch, können aber fachlich adäquat oder inadäquat sein). Diese werden von ihnen allerdings nur wenig bei der Diagnose der Lernendenvorstellungen angewendet. Von der ersten zur zweiten Diagnose ist ein Fortschritt zu erkennen, da die Studierenden sich auf das Formulieren konkreter Lernendenvorstellungen fokussieren und dabei sowohl fachlich adäquate als auch inadäquate Vorstellungen thematisieren. Außerdem wird deutlich, dass sie teilweise zwischen Aussagen der Lernenden und deren Vorstellungen differenzieren. Bei der dritten Diagnose gehen die Studierenden allerdings erneut wie in der ersten Diagnose vor und formulieren defizitorientiert Wissenslücken der Lernenden und nur wenige (adäquate) Vorstellungen. Somit ähneln sie in ihrer Vorgehensweise den Studierenden der Gruppe 1.

Ferner finden sich auch hier ähnliche Widersprüche wie bei Gruppe 1 in den Aussagen: So nennen die Studierenden der Gruppe 2 einerseits die Bedeutsamkeit einer Diagnose von Lernendenvorstellungen, was sie auch mit ihren eigenen Erfahrungen als Schülerinnen belegen und sehr anschaulich beschreiben. Andererseits betonen sie wiederkehrend den hohen Zeitaufwand einer ausführlichen Diagnose von Lernendenvorstellungen ziehen daraus das Fazit, dass eine solche Diagnose im Chemieunterricht in der Schule nicht realisierbar sei. Dies wird außerdem mit unterrichtspraktischen Erfahrungen in schulischen Praktika belegt. Diese Vorstellung, dass eine Diagnose im Unterricht zeitlich nicht durchführbar sei, unterscheidet sie von den beiden anderen Gruppen. Die Studierenden gehen ferner davon aus, dass es sich bei den Lernendenvorstellungen, die sie diagnostiziert haben, um Extrembeispiele handelt, die nicht repräsentativ für den Chemieunterricht sind. Auch hier zeigen sich Eigenheiten in den Vorstellungen.

\section{Identifizierung und Beschreibung bedeutungstragender Situationen}

Die Gruppen 1 und 3 zeigen unterschiedliche Ausgangssituationen und Entwicklungsverläufe hinsichtlich ihrer Vorstellungen über Lernendenvorstellungen sowie hinsichtlich ihrer Diagnosefähigkeiten. Während die erste Gruppe lediglich beim zweiten Fall Anzeichen einer formellen Diagnose erkennen lässt, so zeigt sich bei der dritten Gruppe, dass die Studierenden ab dem zweiten Fall hierzu in der Lage sind. Im Rahmen dieser Studie lassen insbesondere drei Aspekte der Aktivierung reflexiver Prozesse beobachten, die sich als bedeutungstragend in diesem Kontext erweisen können:

\section{Der reflexive Einbezug der eigenen schulischen und hochschulischen Lernbiografie in den Diagnoseprozess}

$\mathrm{Zu}$ diesem Aspekt zählen neben einer Bezugnahme zur eigenen Lernbiografie auch eine Bezugnahme zum aktuellen 
fachchemischen Wissensstand. Beides ist eng verbunden mit einer Identifikation mit den jeweiligen Lernenden in den Vignetten bzw. einer Perspektivübernahme. Während dies den Studierenden der Gruppen 2 und 3 gelingt, zeigen die beiden Studierenden der Gruppe 1 hier keine Anzeichen für eine Perspektivübernahme bzw. eine Identifikation mit den Lernenden. Vielmehr beschreiben sie, dass sie die Aufgabenstellungen zum Teil so wahrgenommen haben, dass sie aus einer Lehrendenperspektive heraus agieren sollen. Auch zeigen sie keinerlei Hinweise darauf, dass sie sich mit den Verstehensprozessen der Lernenden identifizieren können. Die Studierenden der Gruppen 2 und 3 äußern wiederholt, dass sie über vergleichbare (alternative) Vorstellungen verfügten bzw. sich mit den Lernenden in der Vignette identifizieren können.

G3_S3: Ja, aber ich dachte als Kind auch, dass Luft nichts wiegt. (Gruppe 3, 1. Fall)

G2_S1: Feststoff wird flüssig.

G2_S2: Wobei ich glaube das hätte ich auch geschrieben damals in der Schule.

\section{(Gruppe 2, 2. Fall)}

G2_S1: Manchmal muss ich sagen finde ich mich da wieder. Damals als Schülerin halt

G2_S2: Ja, sage ich ja, das hätte auch von mir kommen können damals.

\section{(Gruppe 2, 3. Fall)}

Auch im Antwortverhalten hinsichtlich der Einschätzung des Einflusses ihres Fachwissens auf die Diagnose von Lernendenvorstellungen zeigen die Studierenden ein kohärentes Muster. So beschreiben die Studierenden der Gruppe 3, dass sie bei der Bearbeitung des zweiten Falls ihr eigenes Fachwissen hinterfragt haben. Die Studierenden der Gruppe 1 hingegen geben an, dass ihnen ihr Fachwissen u. a. bei der Identifikation von „fehlerhaften Antworten“ geholfen hat.

\section{Nicht-transmissive bzw. konstruktivistische Perspektive(n) erfahrbar machen: Lernende können unabhängig von ihrer Leistung auch nachunterrichtlich nicht adäquate Konzepte äußern}

Auch bei diesem Aspekt lassen sich die Äußerungen der Studierenden der Gruppen 1 und 3 zwei gegenüberstehenden Polen zuordnen, wenngleich alle Studierenden eine gewisse Überraschung zeigen, dass die Lernenden über nachunterrichtliche alternative Vorstellungen verfügen. Während die Studierenden der Gruppe 1 die alleinige Begründung für die Existenz von nachunterrichtlichen alternativen Vorstellungen bei den Lernenden selbst suchen (s. Tab. 6), nennen die Studierenden der Gruppe 3 u. a. das Ausbleiben eines kognitiven Konflikts bzw. das Nichteingehen der Lehrkraft auf die vorhandenen Vorstellungen als mögliche Gründe (s. Tab. 9). Die Studierenden der ersten Gruppe knüpfen die Notengebung darüber hinaus eng an die vorhandenen fachlichen Vorstellungen und betonen, dass Lernende mit alternativen Vorstellungen bei ihnen im Unterricht über keine guten Noten verfügen würden.

\section{Strukturiertes vorgehen: Diagnoseergebnisse sind mit denen in einer Beispiellösung systematisch zu vergleichen}

Insbesondere bei der dritten Gruppe zeigen sich im Rahmen der ersten Konfrontation mit der Beispiellösung, dass die Studierenden auf einer inhaltlichen sowie einer methodischen Ebene einerseits wichtige Unterschiede feststellen und andererseits zielführende Konsequenzen für ihre weitere Bearbeitung ableiten. So beschreiben sie bspw., dass sie zukünftig insbesondere auch fachlich adäquate Lernendenvorstellungen diagnostizieren werden. Die Ergebnisse der Auswertung des zweiten und des dritten Falls zeigen, dass sie dieses Vorhaben auch umsetzen. Ferner ist dies kongruent mit ihren Vorstellungen über Lernendenvorstellungen und dem Stellenwert einer Diagnose zur Ermittlung einer Lernausgangslage. Bei den anderen beiden Gruppen werden keine entsprechenden Beobachtungen bzw. Änderungen der zukünftigen Vorgehensweise explizit beschrieben. So geben die Studierenden der Gruppe 1 an, dass sie zum Teil massive Unterschiede und nur wenige Übereinstimmungen mit der Beispiellösung feststellen. Teilweise leiten sie auch einige Konsequenzen hieraus ab, befolgen diese aber im weiteren Verlauf nicht - was sie auf Nachfrage auch selbst beschreiben.

\section{Diskussion und Limitationen}

In dieser Studie wurde untersucht, wie angehende Chemielehrkräfte Lernendenvorstellungen diagnostizieren, inwiefern sich ihre diagnostischen Fähigkeiten im Verlauf eines Semesters verändern sowie über welche Vorstellungen die Studierenden hinsichtlich Lernendenvorstellungen verfügen und inwiefern diese sich verändern. Hierfür wurden drei Kleingruppen á 2 bzw. 3 Studierenden über den Zeitraum eines Semesters in einer explorativen Studie begleitet. Aufgrund der Fülle und Komplexität der vorliegenden Daten wurde im Rahmen dieses Beitrags ein Fokus auf zwei der drei Gruppen gelegt, die als Kontrastgruppen hinsichtlich ihrer Entwicklung sowie ihrer Vorstellungen betrachtet werden können.

\section{Fragestellung 1: Vorstellungen über Lernendenvorstellungen}

Die Studierenden verfügen über eine Vielzahl unterschiedlicher Vorstellungen über Lernendenvorstellungen, deren 
Diagnose sowie den weiteren Umgang mit diesen. Die Vorstellungen der Studierenden aus den Gruppen 1 und 3 entsprechen in weiteren Teilen den aus der Literatur (Barthmann et al. 2019; Davis et al. 2006; Gomez-Zwiep 2008; Ilyas und Saeed 2018; Larkin 2012; Mellado 1997; Meyer 2004; Pine et al. 2001) abgeleiteten Positionen Lernendenvorstellungen sind Fehler bzw. Defizite (Gruppe 1) sowie Lernendenvorstellungen sind Ressource bzw. Potenzial (Gruppe 3). Die Vorstellungen der Studierenden über Lernendenvorstellungen bzw. deren Diagnose entwickeln sich im Zuge dieser Studie nur teilweise weiter. So gelingt es der Gruppe 3, ihre Vorstellungen weiter auszubauen. Da diese Vorstellungen analog zu Vorstellungen über Lehr-Lernprozesse als stabil gelten, kann auch dies als literaturkonform angesehen werden. So berichten bspw. Pilitsis und Duncan (2012), dass angehende Lehrkräfte in einem 15-wöchigen Kurs zwar ihre Vorstellungen hin zu stärker konstruktivistischen Vorstellungen entwickeln, gleichzeitig betonen sie jedoch auch, dass die angehenden Lehrkräfte unterschiedlich auf ihre Intervention ansprechen. Auch in weiteren Studien werden positive Effekte von vergleichbar langen Interventionen beschrieben (z. B. Richardson und Kile 1999). Aufbauend auf Postareff et al. (2008b) lässt sich hinzufügen, dass es nicht bei einer Intervention verbleiben darf, da sonst die Gefahr besteht, dass die vorherigen Vorstellungen wieder Verwendung finden. In der Literatur finden sich außerdem Studien, die keinen positiven Effekt feststellen können (z. B. Norton et al. 2005). Ein Grund hierfür kann in der nicht hinreichenden Antizipation der Vorstellungen der (angehenden) Lehrkräfte im Zuge der Planung einer Intervention liegen (Pilitsis und Duncan 2012). Für eine Weiterentwicklung von Vorstellungen im hochschuldidaktischen Bereich sollten daher im Sinne einer konstruktivistischen Lernumgebung typische Studierendenvorstellungen bekannt sein, damit diese Berücksichtigung bei der Planung weiterer Lerngelegenheiten finden können (Klinghammer et al. 2016; Lohmann 2006).

Reflexive Prozesse können bei der Weiterentwicklung der Vorstellungen hilfreich sein (Fischler 2000b). Unterstützt werden können diese Prozesse durch die Verwendung von Leitfragen oder Prompts (Hübner et al. 2007; Schaefer et al. 2012). Insbesondere die Studierenden der Gruppe 1 reflektieren trotz solch eines Einsatzes nur geringfügig ihre Diagnoseergebnisse und ihre Vorstellungen. Fischler (2000a) betont hierzu, dass Studierende Vorstellungen, die ihren bisherigen Erfahrungen als Schülerinnen und Schüler entgegenstehen, häufig ablehnen. Aus dem Bereich der Lernendenvorstellungen ist zusätzlich bekannt, dass Lernende eine Vielzahl von Strategien anwenden, um ihre alternativen Vorstellungen möglichst lange unverändert lassen $\mathrm{zu}$ können (Chinn und Brewer 1998). Abschließend sei auf Rath (2017) verwiesen, die ebenfalls berichtet, dass die Pro- banden in ihrer Studie ihre Diagnoseergebnisse nur selten tiefergehend reflektieren.

\section{Fragestellung 2: Diagnostische Fähigkeiten und deren Entwicklung}

Die Ergebnisse zeigen insgesamt ein gemischtes Bild. So gelingt es nur den Studierenden der Gruppe 3 sich hinsichtlich ihrer Vorgehensweise umfassend weiterzuentwickeln. Bei den Studierenden der Gruppe 1 ist dies lediglich partiell zu beobachten. Alle Studierenden berichten, dass Lernendenvorstellungen eine zentrale Bedeutung für den Lernprozess haben. Demgegenüber steht jedoch bei den Studierenden der Gruppe 1, dass sie nur partiell formelle Diagnosen durchführen. Diese scheinbar widersprüchlichen Ergebnisse werden auch an anderer Stelle berichtet (Cox et al. 2016; Gomez-Zwiep 2008; Mellado et al. 2007; Morrison und Lederman 2003). Mellado et al. (2007) konnten in ihrer Studie bspw. einen solch diametralen Unterschied zwischen geplantem und tatsächlichem Verhalten im Umgang mit Lernendenvorstellungen bei Lehrkräften beobachten. Auch Barnhart und van Es (2015) und Larkin (2012) betonen, dass ein Bewusstsein für die Bedeutung von Lernendenvorstellungen noch nicht garantiert, dass die Vorstellungen auch wirklich analysiert werden.

Den Studierenden gelang es nur in Teilen, zentrale (häufige) Lernendenvorstellungen als solche zu identifizieren. Yang et al. (2014) berichten im Rahmen ihrer Studie ebenfalls, dass es Lehrkräften schwerfällt, häufige von weniger häufigen Lernendenvorstellungen zu unterscheiden. Bedingt durch diese Wissenslücken wenden Lehrkräften, Yang et al. (2014) zufolge, informelle Diagnosemethoden (z.B. Ausschlussverfahren) an, um die Bedeutung von Lernendenvorstellungen vorherzusagen. Ein ähnliches Verhalten lässt sich bei den Studierenden der Gruppe 1 beobachten. Auch hier finden sich insbesondere in den Bearbeitungen des ersten und dritten Falls zahlreiche Elemente einer informellen Diagnose. Auffällig ist weiterhin, dass es den Studierenden der Gruppe 3 gelingt, einen Perspektivwechsel durchzuführen und sie sich mit den Lernenden in der Vignette und ihren Lernprozessen identifizieren. Die Studierenden der Gruppe 1 führen dies nicht durch. Alonzo und Kim (2015) berichten einen ähnlichen Zusammenhang zwischen dem Ausbleiben einer Perspektivübernahme und einer schwach ausgebildeten Wahrnehmung von Lernendenvorstellungen.

Hinsichtlich der Unterstützung von diagnostischen Prozessen werden in der Literatur unterschiedliche prozessbezogene Modelle bzw. Abläufe vorgeschlagen (z.B. Beretz et al. 2017). In dieser Studie wurde den Studierenden keine Abfolge an Schritten vorgegeben. Derartige Scaffolds können sich jedoch als hilfreich für die Bearbeitung diagnostischer Aufgaben erweisen (Beretz et al. 2017). 
Abschließend sei festgehalten, dass sich die Diagnoseergebnisse der jeweiligen Studierendengruppen fallspezifisch unterscheiden. Dies kann einerseits auf einen Effekt der Studie zurückgeführt werden. Andererseits berichten auch Rath (2017) bzw. Hoppe et al. (2020), dass die Diagnose von Lernendenvorstellungen kontext- bzw. situationsspezifisch ausfallen kann. Hier können das jeweilige Fachwissen bzw. fachdidaktische Wissen eine Auswirkung haben (s. unten).

\section{Fragestellung 3: Beschreibung bedeutungstragender Situationen}

Im Zusammenhang mit den unterschiedlichen Entwicklungsprozessen können unterschiedliche Handlungen und Reflexionsprozesse beobachtet werden. Insbesondere die Reflexion der eigenen Lehr-Lernbiografie kann mit einer positiven Entwicklung assoziiert werden. Aufgrund der geringen Anzahl an untersuchten Gruppen sowie der Ausrichtung der Studie sind allgemeingültige Aussagen nicht ableitbar. Auch ist es als wahrscheinlich anzusehen, dass es weitere und indifferentere Entwicklungsverläufe bei weiteren Studierenden gibt. Jedoch stehen die Ergebnisse der hier dargestellten zwei Extremgruppen im Einklang mit weiteren Studienergebnissen. So betont bspw. Jelemenska (2012), dass eine intensive Auseinandersetzung mit den eigenen Erfahrungen zentral für die Weiterentwicklung der individuellen Vorstellungen hinsichtlich Lernendenvorstellungen ist. Auch Park und Oliver (2008) machen deutlich, dass eine potentialorientierte Haltung gegenüber Lernendenvorstellungen eine zentrale Voraussetzung für eine Weiterentwicklung des fachdidaktischen Wissens sei.

\section{Gruppenzusammensetzung und Einfluss des Designs}

Die Studierenden der Gruppe 3 haben im Vergleich mit den beiden anderen Gruppen jeweils zwei weitere chemiedidaktische Veranstaltungen besucht. Dies kann dazu führen, dass sie über stärker konstruktivistische Vorstellungen verfügen, die im Laufe des Studiums weiterentwickeln wurden (Brauer et al. 2008). Ferner ist denkbar, dass sie innerhalb dieser Veranstaltungen mit weiteren Lernangeboten konfrontiert waren, was sich ebenfalls positiv auf das Wissen um Lernendenvorstellungen auswirken kann (Barthmann 2018; Pietzner 2015). Es gibt allerdings auch Argumente, nach denen kein positiver Einfluss bestehen muss. Erstens gelten Vorstellungen über Lehren und Lernen sowie über Lernendenvorstellungen als stabil (Norton et al. 2005). Zweitens existiert in der Literatur hinsichtlich des Umgangs mit Lernendenvorstellungen kein eindeutig positiver Zusammenhang mit der Berufserfahrung (Diakidoy und Iordanou 2003; Gomez-Zwiep 2008; Yang et al. 2014).
Das Design dieser Studie ist explorativ ausgelegt. Dementsprechend wurden qualitative Forschungsmethoden auf eine geringe Anzahl an Probandinnen und Probanden angewendet. Somit können weder verallgemeinerungsfähige Aussagen über die diagnostischen Fähigkeiten noch über deren Entwicklung oder einen potenziellen Zusammenhang zwischen den Vorstellungen der Studierenden und ihren diagnostischen Fähigkeiten getroffen werden. Hierfür wäre bspw. ein Kontrollgruppendesign zielführend gewesen, welches u. a. bei Hoppe et al. (2020) zu finden ist.

Diagnostische Prozesse im Unterrichtsgeschehen sind hochgradig komplex (Heitzmann et al. 2019). Zu Zwecken der Förderung diagnostischer Fähigkeiten ist der Einsatz von unterrichtsrealistischen Lernumgebungen daher weniger zielführend (Wildgans-Lang et al. 2020). Vielmehr ist eine Reduktion dieser Komplexität bei gleichzeitiger Fokussierung auf einzelne Inhalte bzw. Lernende notwendig (Dannemann et al. 2019; Heitzmann et al. 2019; WildgansLang et al. 2020). Larkin (2012) zufolge ist die Fähigkeit Lernendenvorstellungen zu antizipieren und ihren Ursprung $\mathrm{zu}$ verstehen eine grundlegende Voraussetzung, um mit ihnen im späteren Berufsalltag umzugehen. In dieser Studie wurden daher Videovignetten im Rahmen des fallbasierten Lernens eingesetzt, die nur wenige Schülerinnen und Schüler bei der Auseinandersetzung mit einem fachlichen Inhalt zeigen. Nach Seifried und Wuttke (2017) bereitet ein Einsatz von komplexitätsreduzierten Situationen angehende Lehrkräfte nicht notwendigerweise auf das spätere Unterrichtsgeschehen vor. Es bleibt offen, ob bzw. inwiefern die Studierenden der drei Gruppen in realen Praxissituationen im Studium oder im späteren Unterricht weiterhin so agieren. Fischler (2000a) betont, dass der Praxisdruck in solchen Situationen zu einem falsch aufgefassten Pragmatismus führen kann, der in einem lehrkraftzentrierten Unterricht mündet. Der Prozess der Diagnose im Unterrichtsgesehen besteht darüber hinaus aus einer komplexen Interaktion zwischen beurteilender und beurteilter Person. Diese Interaktion kann sich auf den Prozess der Diagnose auswirken (Ingenkamp und Lissmann 2008). Daher sind Erweiterungen der Lernumgebungen um z.B. Anteile in Lehr-Lernlaboren als zielführende Maßnahme anzusehen.

\section{Implikationen und Ausblick}

Bereits seit den 1980er-Jahren werden das Wissen und die Fähigkeiten von (angehenden) Lehrkräften hinsichtlich Lernendenvorstellungen untersucht (Nussbaum 1981). Über die vergangenen Jahrzehnte werden jedoch kaum positive Veränderungen beschrieben (Cox et al. 2016; Diakidoy und Iordanou 2003; Gomez-Zwiep 2008; Ilyas und Saeed 2018; Larkin 2012; Mellado 1997; Meyer 2004; Morrison und Lederman 2003; Naah 2015; Nussbaum 1981; Pine et al. 
2001; Yang et al. 2014). So finden sich in der aufgeführten Literatur zahlreiche Berichte darüber, dass das Wissen von (angehenden) Lehrkräften über Lernendenvorstellungen gering ist, sie diese eher unterschätzen oder selbst über alternative Vorstellungen verfügen. Cox et al. (2016) heben in diesem Zusammenhang hervor, dass bedingt durch die Unterschätzung von Lernendenvorstellungen, Lehrkräfte keinerlei Notwendigkeit sehen, ihren Unterricht anzupassen. Als weitere Gründe für diese Schwierigkeiten werden einerseits fehlende Lerngelegenheiten innerhalb der universitären Bildung angesprochen (Gomez-Zwiep 2008; Marohn und Rohrbach 2013). Andererseits wird kritisiert, dass trotz umfangreicher Erkenntnisse aus jahrzehntelanger Forschung nur wenig praxisnahe bzw. direkt anwendbare Umsetzungen existieren (Pine et al. 2001; Yang et al. 2014). Auch an anderer Stelle wird die Bedeutung der universitären Bildung zum Aufbau diagnostischer Fähigkeiten als zentral hervorgehoben (Cappell 2013; Cox et al. 2016; Ilyas und Saeed 2018; Lucero et al. 2017; Naah 2015). Selbst Lehrkräfte betonten dies in Befragungen (Barthmann 2018). Analog zu Fördermöglichkeiten im schulischen Kontext, sollten auch universitäre Bildungsangebote an den Lernvoraussetzung und insbesondere den Vorstellungen der Studierenden ansetzen (Lohmann 2006; van Dijk und Kattmann 2010).

Im Rahmen dieses Beitrags wurden anhand der vertiefenden Analysen von Studierendenkleingruppen Hinweise abgleitet, die bei der Gestaltung fachdidaktischer Interventionen berücksichtigt werden können (s. hierzu auch Fischler 2000b). Eine mögliche Deutung dieser Studie ist, dass Fördermaßnahmen weniger effektiv sind, solange die Studierenden ihre Vorstellungen über Lernendenvorstellungen nicht weiterentwickelt haben. Auch Postareff et al. (2008b) betonen, dass im Rahmen von Bildungsangeboten primär eine Erweiterung der bestehenden Vorstellungen das Ziel sein sollte und nicht eine Veränderung der Lehrtechniken. Um dies zu erreichen, sollten u. a. Reflexionsprozesse angestoßen werden, in denen die Studierenden sich kritisch mit ihrer eigenen Lehr-Lernbiografie auseinandersetzen (s. hierzu auch Fischer et al. 2003). Abschließend sei darauf verwiesen, dass hierfür eine Vielzahl an unterschiedlichen Lerngelegenheiten benötigt werden (Cappell 2013).

Als weitere Implikation lässt sich im Hinblick auf eine gezielte Theorie-Praxis-Verbindung innerhalb der universitären fachdidaktischen Lehre ableiten, dass Videobeispiele guter Praxis zur Diagnose und zum Umgang mit Lernendenvorstellungen im Unterricht benötigt werden. Einerseits könnten derartige Videos als überzeugende Lerngelegenheiten dienen, um bspw. die zeitliche und organisatorische Durchführbarkeit zu demonstrieren. Andererseits könnten sie auch als Ausgangpunkt genutzt werden, um theoretische
Conceptual Change Ansätze hieran zu erforschen (s. hierzu auch Blomberg et al. 2013).

von Aufschnaiter und Alonzo (2018) betonen, dass für die Analyse der Verstehensprozesse von Lernenden (z.B. dargestellt in Videovignetten) dringend ein theoretischer Analyserahmen vorliegen und angewendet werden sollte. Hier zeigt sich eine größere Herausforderung insbesondere für die Chemiedidaktik: Während für viele Themen bzw. Kontexte die Existenz und Häufigkeit von Lernendenvorstellungen gut dokumentiert ist (z. B. Bain und Towns 2016; Heeg et al. 2020a; Grüß-Niehaus und Schanze 2011; Özmen 2004), werden nur in wenigen Fällen die in der Literatur vorhandenen Theorien zur Entstehung bzw. Strukturierung von Vorstellungen (Amin 2009; Chi 2013; diSessa 2008; Vosniadou 2013) zur näheren Beschreibung angewendet (Ausnahmen z.B. bei Chiu et al. 2002; Lin und Chiu 2007). Gleiches gilt für die Gestaltung von Lernprozessen. Auch hier finden sich bislang wenige Beispiele innerhalb der Chemiedidaktik, die einen der bestehenden Ansätze zur theoriegeleiteten Entwicklung von Lernangeboten nutzen. Daher kann nach Auffassung der Autoren im Rahmen der Chemiedidaktik aktuell zwar von einer empiriebasierten aber noch nicht von einer theoriebasierten Diagnose gesprochen werden. Diese Variante richtet sich entsprechend nach der Häufigkeit der Lernendenvorstellungen aus bzw. nutzt für die Förderung bereits erprobte Lernangebote. Der conceptual metaphor Ansatz (Daane et al. 2018; Niebert et al. 2012; Niebert und Gropengießer 2015) sowie der Ansatz der learning progression (Alonzo und von Aufschnaiter 2018; Emden et al. 2018) sind zwei recht vielversprechende Varianten, die teilweise bereits in Fortbildungen von Lehrkräften als hilfreich in der Anwendung beschrieben werden (Daane et al. 2018).

In dieser Studie wurden, ähnlich wie bei Rath (2017), Performanzdaten erhoben. Aus diesen Performanzdaten wurde auf die zugrundeliegenden diagnostischen Fähigkeiten der Studierenden geschlossen. Ferner wurden mit den Vorstellungen der Studierenden über Lernendenvorstellungen ein möglicher Einflussfaktor (eine Disposition) identifiziert. Auch, wenn Verbindungen zwischen diesen Daten in Teilen hergestellt wurden, so fand dennoch eine getrennte Analyse statt. Derartige Daten können allerdings auch Rückschlüsse auf eine zugrundeliegende Diagnosekompetenz liefern (Herppich et al. 2017). In der pädagogischen wie fachdidaktischen Literatur finden sich viele Studien, die von einer Diagnosekompetenz ausgehen und diese mehr oder weniger explizit beschreiben (Abs 2007; von Aufschnaiter et al. 2015; Dannemann et al. 2018; Heinrichs 2015; Heitzmann et al. 2019; Herppich et al. 2017; Hofman und Roth 2018; Rath 2017; Wedel et al. 2019). Ein vielversprechendes Modell zur Beschreibung von Kompetenzen im Rahmen der Lehrkräftebildung ist das competence-ascontinuum-Modell von Blömeke et al. (2015). In dieser 
Modellierung werden Dispositionen, situationsspezifische Fähigkeiten (u.a. noticing) sowie die Performanz, also das beobachtbare Verhalten, als ein Kontinuum beschrieben. Dieses Modell kann auch für den vorliegenden Fall einen geeigneten Rahmen bilden. Mögliche Dispositionen wären dann bspw. das fachdidaktische sowie das fachliche Wissen (Baumert und Kunter 2006). Insbesondere das Wissen um (häufige) alternative Lernendenvorstellungen wird hervorgehoben (Herppich et al. 2017). Weitere Studien lassen jedoch den Schluss zu, dass es keine einfachen Zusammenhänge zwischen dem fachlichen Wissen (Rath 2017) sowie dem fachdidaktischen Wissen (McElvany et al. 2009) gibt. Rieu et al. (2020) führen diese Ergebnisse teilweise auf die Erhebungen zurück und betonen, dass die jeweiligen Wissensfacetten situationsspezifisch erhoben werden sollten. $\mathrm{Zu}$ den weiteren Leistungsdispositionen kann auch das Wissen um eine formelle bzw. informelle Diagnose gehören (Herppich et al. 2017). Die Ergebnisse dieser Studie legen außerdem nahe, dass zu den weiteren Dispositionen - wie oben bereits erwähnt - auch die Vorstellungen über die Diagnose sowie den Gegenstand der Diagnose zählen können. Dies steht im Einklang mit Annahmen von Herppich et al. (2017). Ein zentraler Bereich, der zumindest für die Diagnose von Lernendenvorstellungen wenig erforscht ist, ist der der situationsspezifischen Fähigkeiten. Hier spielen Aspekte, wie bspw. noticing (Barnhart und van Es 2015; Sherin und van Es 2005; van Es und Sherin 2008) eine zentrale Rolle. Inwiefern diese Aspekte bei der Diagnose von Lernendenvorstellungen in komplexitätsreduzierten Settings, wie sie hier zum Einsatz kamen, eine Rolle spielen, kann ein lohnenswerter Gegenstand künftiger Forschung sein. Im Rahmen dieser Studie wurden die diagnostischen Fähigkeiten weniger Studierende in einer explorativen Untersuchung über den Zeitraum eines Semesters beobachtet. Daher sind die Ergebnisse nicht generalisierbar bzw. lassen sich nicht auf andere Kontexte übertragen (Johnson und Onwuegbuzie 2004). Es können jedoch wertvolle Hinweise für kommende Studien abgeleitet werden. Diesen Hinweisen sollten in kommenden Untersuchungen auch quantitativ nachgegangen werden. Das hier verwendete Kategoriensystem erweist sich als tragfähig und ausbaufähig, um erhobene Performanzdaten zu bewerten. Diese können anschließend auch Verwendung im Rahmen eines Diagnosekompetenzmodells finden (Herppich et al. 2017).

Abschließend sei auf einen interessanten Vergleich hingewiesen, den Heitzmann et al. (2019) sowie Chernikova et al. (2020) zwischen den Tätigkeitsfeldern Medizin und Pädagogik in Hinblick auf die Diagnose ziehen. So sind (alternative) Lernendenvorstellungen selbstredend keine Krankheit und sollten auch nicht als solche angesehen werden (Larkin 2012). Allerdings ist die medizinische Diagnostik ein umfangreiches theorie- und empiriebasiertes System. Es existieren zu einer Vielzahl von Krankheiten und Krankheitsbildern Leitlinien, die die Diagnostik erleichtern und gleichzeitig standardisierte Handlungsempfehlungen formulieren (Kopp et al. 2002; Perleth et al. 2000). Es gibt demnach gute Gründe, auch für einzelne Themen theorie- und empiriebasierte sowie gleichermaßen praxisorientierte Leitlinien für den Umgang mit Lernendenvorstellungen zu entwickeln und zu evaluieren. Dies sollte jedoch nicht für Lehrkräfte, sondern vielmehr mit Lehrkräften zusammen gesehen. Denn auch die besten Leitlinien müssen in der Praxis Verwendung finden und dort bestehen - auch dies lässt sich aus dem Bereich der Medizin lernen (Lelgemann und Ollenschläger 2006).

Danksagung Diese Studie entstand im Rahmen des Projektes „Theoria cum praxi. Förderung von Reflektierter Handlungsfähigkeit als Leibniz-Prinzip der Lehrerbildung“ (Förderkennzeichen 01JA1506), das aus Mitteln des Bundesministeriums für Bildung und Forschung im Rahmen der „Qualitätsoffensive Lehrerbildung“ gefördert wird. Wir bedanken uns bei allen Studierenden, die durch ihre Teilnahme dieses Projekt ermöglicht haben. Auch möchten wir uns bei den Gutachterinnen bzw. Gutachtern herzlich für ihre wertvollen Rückmeldungen bedanken, die zu einer deutlichen Weiterentwicklung dieses Artikels geführt haben.

Funding Open Access funding enabled and organized by Projekt DEAL.

Open Access Dieser Artikel wird unter der Creative Commons Namensnennung 4.0 International Lizenz veröffentlicht, welche die Nutzung, Vervielfältigung, Bearbeitung, Verbreitung und Wiedergabe in jeglichem Medium und Format erlaubt, sofern Sie den/die ursprünglichen Autor(en) und die Quelle ordnungsgemäß nennen, einen Link zur Creative Commons Lizenz beifügen und angeben, ob Änderungen vorgenommen wurden.

Die in diesem Artikel enthaltenen Bilder und sonstiges Drittmaterial unterliegen ebenfalls der genannten Creative Commons Lizenz, sofern sich aus der Abbildungslegende nichts anderes ergibt. Sofern das betreffende Material nicht unter der genannten Creative Commons Lizenz steht und die betreffende Handlung nicht nach gesetzlichen Vorschriften erlaubt ist, ist für die oben aufgeführten Weiterverwendungen des Materials die Einwilligung des jeweiligen Rechteinhabers einzuholen.

Weitere Details zur Lizenz entnehmen Sie bitte der Lizenzinformation auf http://creativecommons.org/licenses/by/4.0/deed.de.

\section{Literatur}

Abs, H.J. (2007). Überlegungen zur Modellierung diagnostischer Kompetenz bei Lehrerinnen und Lehrern. In M. Lüders (Hrsg.), Forschung zur Lehrerbildung. Kompetenzentwicklung und Programmevaluation (S. 63-84). Münster u. a.: Waxmann.

Akaygun, S., \& Jones, L.L. (2014). Words or Pictures. A comparison of written and pictorial explanations of physical and chemical equilibria. International Journal of Science Education, 36(5), 783-807. https://doi.org/10.1080/09500693.2013.828361.

Al-Amoush, S., Markic, S., Usak, M., Erdogan, M., \& Eilks, I. (2014). Beliefs about chemistry teaching and learning-A comparison of teachers' and student teachers' beliefs from Jordan, Turkey and Germany. International Journal of Science and Mathematics Education, 12(4), 767-792. https://doi.org/10.1007/s10763-0139435-7.

Alonzo, A.C., \& von Aufschnaiter, C. (2018). Moving beyond misconceptions: learning progressions as a lens for seeing progress 
in student thinking. The Physics Teacher, 56(7), 470-473. https:// doi.org/10.1119/1.5055332.

Alonzo, A.C., \& Kim, J. (2015). Declarative and dynamic pedagogical content knowledge as elicited through two video-based interview methods. Journal of Research in Science Teaching. https://doi.org/ 10.1002/tea.21271.

Amin, T.G. (2009). Conceptual metaphor meets conceptual change. Human Development, 52(3), 165-197. https://doi.org/10.1159/ 000213891.

Artelt, C., \& Gräsel, C. (2009). Diagnostische Kompetenz von Lehrkräften. Zeitschrift für pädagogische Psychologie, 23(34), 157-160. https://doi.org/10.1024/1010-0652.23.34.157.

Atria, M., Strohmeier, D., \& Spiel, C. (2006). Der Einsatz von Vignetten in der Programmevaluation. Beispiele aus dem Anwendungsfeld „Gewalt in der Schule“. In U. Flick (Hrsg.), Qualitative Evaluationsforschung. Konzepte - Methoden - Umsetzung. rororo Rowohlts Enzyklopädie, (Bd. 55674, S. 233-249). Reinbek bei Hamburg: Rowohlt-Taschenbuch-Verl.

von Aufschnaiter, C., \& Alonzo, A.C. (2018). Foundations of formative assessment: Introducing a learning progression to guide preservice physics teachers' video-based interpretation of student thinking. Applied Measurement in Education, 31(2), 113-127. https:// doi.org/10.1080/08957347.2017.1408629.

von Aufschnaiter, C., \& Rogge, C. (2010). Misconceptions or missing conceptions? EURASIA Journal of Mathematics, Science \& Technology Education, 6(1), 3-18.

von Aufschnaiter, C., Cappell, J., Dübbelde, G., Ennemoser, M., Mayer, J., Stiensmeier-Pelster, J., Sträßer, R., \& Wolgast, A. (2015). Diagnostische Kompetenz. Theoretische Überlegungen zu einem zentralen Konstrukt der Lehrerbildung. Zeitschrift für Pädagogik, 5, 738-758.

von Aufschnaiter, C., Selter, C., \& Michaelis, J. (2017). Nutzung von Vignetten zur Entwicklung von Diagnose- und Förderkompetenzen. Konzeptionelle Überlegungen und Beispiele aus der MINTLehrerbildung. In C. Selter, S. Hußmann, C. Hößle, C. Knipping, K. Lengnink \& J. Michaelis (Hrsg.), Diagnose und Förderung heterogener Lerngruppen. Theorien, Konzepte und Beispiele aus der MINT-Lehrerbildung (S. 85-105). Münster: Waxmann.

Baalmann, W., Frerichs, V., Weitzel, H., Gropengießer, H., \& Kattmann, U. (2004). Schülervorstellungen zu Prozessen der Anpassung - Ergebnisse einer Interviewstudie im Rahmen der Didaktischen Rekonstruktion. Zeitschrift für Didaktik der Naturwissenschaften, 10, 7-28.

Bain, K., \& Towns, M.H. (2016). A review of research on the teaching and learning of chemical kinetics. Chemistry Education Research and Practice, 17(2), 246-262. https://doi.org/10.1039/ C5RP00176E.

Barnhart, T., \& van Es, E. A. (2015). Studying teacher noticing: examining the relationship among pre-service science teachers' ability to attend, analyze and respond to student thinking. Teaching and Teacher Education, 45, 83-93. https://doi.org/10.1016/j.tate. 2014.09.005.

Bartel, M.-E., \& Roth, J. (2017). Diagnostische Kompetenz von Lehramtsstudierenden fördern. In J. Leuders, T. Leuders, S. Prediger \& S. Ruwisch (Hrsg.), Mit Heterogenität im Mathematikunterricht umgehen lernen. Konzepte und Studien zur Hochschuldidaktik und Lehrerbildung Mathematik, (Bd. 24, S. 43-52). Wiesbaden: Springer.

Barthmann, K. (2018). Vorstellungen von Geographielehrkräften über Schülervorstellungen und den Umgang mit ihnen in der Unterrichtspraxis. Dissertation. Bayreuth: Universität.

Barthmann, K., Conrad, D., \& Obermaier, G. (2019). Vorstellungen von Geographielehrkräften über Schülervorstellungen und den Umgang mit ihnen in der Unterrichtspraxis. Zeitschrift für Geographiedidaktik, 47(3), 78-97. https://doi.org/10.18452/21265.

Baumert, J., \& Kunter, M. (2006). Stichwort: Professionelle Kompetenz von Lehrkräften. Zeitschrift für Erziehungswissenschaft, 9(4), 469-520.
Beretz, A.-K., Lengnink, K., \& von Aufschnaiter, C. (2017). Diagnostische Kompetenz gezielt fördern. Videoeinsatz im Lehramtsstudium Mathematik und Physik. In C. Selter, S. Hußmann, C. Hößle, C. Knipping, K. Lengnink \& J. Michaelis (Hrsg.), Diagnose und Förderung heterogener Lerngruppen. Theorien, Konzepte und Beispiele aus der MINT-Lehrerbildung (S. 149-168). Münster: Waxmann.

Berliner, D.C. (2001). Learning about and learning from expert teachers. International Journal of Educational Research, 35(5), 463-482. https://doi.org/10.1016/S0883-0355(02)00004-6.

Blomberg, G. A., Renkl, A., Gamoran Sherin, M., Borko, H., \& Seidel, T. (2013). Five research-based heuristics for using video in preservice teacher education. Journal für Bildungsforschung Online, 5(1), 90-114.

Blomberg, G. A., Sherin, M.G., Renkl, A., Glogger, I., \& Seidel, T. (2014). Understanding video as a tool for teacher education. Investigating instructional strategies to promote reflection. Instructional Science, 42(3), 443-463. https://doi.org/10.1007/s11251013-9281-6.

Blömeke, S., Gustafsson, J.-E., \& Shavelson, R. J. (2015). Beyond dichotomies. Zeitschrift für Psychologie, 223(1), 3-13. https://doi. org/10.1027/2151-2604/a000194.

Borko, H., Jacobs, J., Eiteljorg, E., \& Pittman, M.E. (2008). Video as a tool for fostering productive discussions in mathematics professional development. Teaching and Teacher Education, 24(2), 417-436. https://doi.org/10.1016/j.tate.2006.11.012.

Brauer, H., Balster, S., \& Wilde, M. (2008). Lehr- und Lernvorstellungen künftig Lehrender zum Lernen von Schülerinnen und Schülern im Fach Biologie. Zeitschrift für Didaktik der Naturwissenschaften, 20(1), 191-200. https://doi.org/10.1007/s40573014-0019-z.

Brophy, J. (Hrsg.). (2004). Using video in teacher education. Oxford: Elsevier.

Brovelli, D. (2014). Integrierte naturwissenschaftliche Lehrerbildung - Entwicklung professioneller Kompetenz bei Lehramtsstudierenden. Zeitschrift für Didaktik der Naturwissenschaften, 20(1), 21-32.

Brunner, M., Kunter, M., Krauss, S., Baumert, J., Blum, W., Dubberke, T., Jordan, A., Klusmann, U., Tsai, Y.-M., \& Neubrand, M. (2006). Welche Zusammenhänge bestehen zwischen dem fachspezifischen Professionswissen von Mathematiklehrkräften und ihrer Ausbildung sowie beruflichen Fortbildung? Zeitschrift für Erziehungswissenschaft, 9(4), 521-544. https://doi.org/10.1007/ s11618-006-0166-1.

Bryan, L. A. (2012). Research on science teacher beliefs. In B. J. Fraser, K. Tobin \& C. J. Mcrobbie (Hrsg.), Second international handbook of science education (S. 477-495). Dordrecht: Springer.

Cain, M. (2012). Beliefs about classroom practice. A study of primary teacher trainees in Trinidad and Tobago. International Journal of Humanities and Social Science, 2(3), 96-105.

Campbell, P. B. (1996). How would I handle that? Using vignettes to promote good math and science education

Cappell, J. (2013). Fachspezifische Diagnosekompetenz angehender Physiklehrkräfte in der ersten Ausbildungsphase. Studien zum Physik- und Chemielernen, Bd. 146. Berlin: Logos.

Carter, K., Cushing, K., Sabers, D., Stein, P., \& Berliner, D. (1988). Expert-novice differences in perceiving and processing visual classroom information. Journal of Teacher Education, 39(3), 25-31. https://doi.org/10.1177/002248718803900306.

Chandrasegaran, A.L., Treagust, D.F., \& Mocerino, M. (2008). An Evaluation of a Teaching Intervention to Promote Students' Ability to Use Multiple Levels of Representation When Describing and Explaining Chemical Reactions. Research in Science Education, 38(2), 237-248.

Chen, C., Sonnert, G., Sadler, P. M., \& Sunbury, S. (2020). The impact of high school life science teachers' subject matter knowledge and knowledge of student misconceptions on students' learning. 
CBE life sciences education, 19(1), ar9. https://doi.org/10.1187/ cbe.19-08-0164.

Cheng, A. Y.N., Tang, S. Y.F., \& Cheng, M.M.H. (2016). Changing conceptions of teaching: a four-year learning journey for student teachers. Teachers and Teaching, 22(2), 177-197. https://doi.org/ 10.1080/13540602.2015.1055437.

Chernikova, O., Heitzmann, N., Fink, M.C., Timothy, V., Seidel, T., \& Fischer, F. (2020). Facilitating diagnostic competences in higher education - a meta-analysis in medical and teacher education. Educ Psychol Rev, 32(1), 157-196. https://doi.org/10.1007/ s10648-019-09492-2.

Chi, M.T.H. (2013). Two kinds and four sub-types of misconcieved knowledge, ways to change it, and learning outcomes. In S. Vosniadou (Hrsg.), International Handbook of Research on Conceptual Change (S. 49-70). Hoboken: Taylor \& Francis.

Chinn, C. A., \& Brewer, W.F. (1998). An empirical test of a taxonomy of responses to anomalous data in science. Journal of Research in Science Teaching, 35(6), 623-654. https://doi.org/10.1002/(SICI) 1098-2736(199808)35:6〈623::AID-TEA3〉3.0.CO;2-O.

Chiu, M.-H., Chou, C.-C., \& Liu, C.-J. (2002). Dynamic processes of conceptual change: analysis of constructing mental models of chemical equilibrium. Journal of Research in Science Teaching, 39(8), 688-712. https://doi.org/10.1002/tea.10041.

Colestock, A., \& Sherin, M. G. (2009). Teachers' sense-making strategies while watching video of mathematics instruction. Journal of Technology and Teacher Education, 17(1), 7-29.

Coll, R.K., \& Treagust, D.F. (2003). Investigation of secondary school, undergraduate, and graduate learners' mental models of ionic bonding. Journal of Research in Science Teaching, 40(5), 464-486. https://doi.org/10.1002/tea.10085.

Cox, M., Steegen, A., \& de Cock, M. (2016). How aware are teachers of students' misconceptions in astronomy? A qualitative analysis in Belgium. Science education international, 27(2), 277-300.

Daane, A. R., Haglund, J., Robertson, A.D., Close, H.G., \& Scherr, R.E. (2018). The pedagogical value of conceptual metaphor for secondary science teachers. Science \& Education, 102(5), 1051-1076. https://doi.org/10.1002/sce.21451.

Dannemann, S., Heeg, J., \& Schanze, S. (2019). Fallbasierte Förderung der Diagnose- und Planungsfähigkeiten von Lehramtsstudierenden. Lernen mit Videovignetten in der Biologie- und Chemiedidaktik. In E. Christophel, M. Hemmer, F. Korneck, T. Leuders \& P. Labudde (Hrsg.), Fachdidaktische Forschung zur Lehrerbildung 1. Aufl. Fachdidaktische Forschungen, (Bd. 11, S. 75-85). Münster: Waxmann.

Dannemann, S., Meier, M., Hilfert-Rüppell, D., Kuhlemann, B., Eghtessad, A., Höner, K., Hößle, C., \& Looß, M. (2018). Erhebung und Fördern der Diagnosekompetenz von Lehramtsstudierenden durch den Einsatz von Vignetten. In M. Hammann \& M. Lindner (Hrsg.), Biologiedidaktik als Wissenschaft. 21. Internationale Tagung der Fachsektion Didaktik der Biologie im VBIO, Martin-Luther-Universität Halle-Wittenberg, 2017. Lehrund Lernforschung in der Biologiedidaktik, (Bd. 8, S. 245-265). Innsbruck: Studien Verlag.

Davis, E. A., Petish, D., \& Smithey, J. (2006). Challenges new science teachers face. Review of Educational Research, 76(4), 607-651.

Diakidoy, I.-A. N., \& Iordanou, K. (2003). Preservice teachers' and teachers' conceptions of energy and their ability to predict pupils' level of understanding. European Journal of Psychology of Education, 18(4), 357-368. https://doi.org/10.1007/BF03173241.

van Dijk, E. M., \& Kattmann, U. (2010). Evolution im Unterricht: Eine Studie über fachdidaktisches Wissen von Lehrerinnen und Lehrern. Zeitschrift für Didaktik der Naturwissenschaften, 16, 7-21.

diSessa, A.A. (2008). A bird'2-eye view of the "pieces" vs. "coherence" controversy. (from the "pieces" side of the fence). In S. Vosniadou (Hrsg.), International handbook of research on conceptual change. Educational psychology handbook series. (S. 35-60). New York: Routledge.
Döring, N., \& Bortz, J. (2016). Forschungsmethoden und Evaluation in den Sozial- und Humanwissenschaften (5. Aufl.). Springer-Lehrbuch. Berlin: Springer.

Dübbelde, G. (2013). Diagnostische Kompetenzen angehender Biologie-Lehrkräfte im Bereich der naturwissenschaftlichen Erkenntnisgewinnung. Dissertation. Kassel: Universität. https://d-nb.info/ $1046712527 / 34$

Duit, R. (2009). Bibliography-students' and teachers' conceptions and science education

Duit, R., Treagust, D.F., \& Widodo, A. (2008). Teaching science for conceptual change. Theory and practice. In S. Vosniadou (Hrsg.), International handbook of research on conceptual change. Educational psychology handbook series. (S. 629-646). New York: Routledge.

Duit, R., Treagust, D.F., \& Widodo, A. (2013). Teaching science for conceptual change. In S. Vosniadou (Hrsg.), International handbook of research on conceptual change (S. 487-503). Hoboken: Taylor \& Francis.

Ekiz-Kiran, B., \& Boz, Y. (2020). Interactions between the science teaching orientations and components of pedagogical content knowledge of in-service chemistry teachers. Chemistry Education Research and Practice, 21(1), 95-112. https://doi.org/10. 1039/C9RP00092E.

Emden, M., Weber, K., \& Sumfleth, E. (2018). Evaluating a learning progression on 'Transformation of Matter' on the lower secondary level. Chemistry Education Research and Practice, 19(4), 1096-1116. https://doi.org/10.1039/C8RP00137E.

Erickson, F. (2011). On noticing teacher noticing. In M. Sherin, V. Jacobs \& R. Philipp (Hrsg.), Mathematics teacher noticing (S. 17-34). New York, London: Routledge.

van Es, E. A., \& Sherin, M. G. (2008). Mathematics teachers' "learning to notice" in the context of a video club. Teaching and Teacher Education, 24(2), 244-276. https://doi.org/10.1016/j.tate.2006. 11.005 .

Fensham, P. J., Gunstone, R. F., \& White, R. T. (2013). The content of science: a constructivist approach to its teaching and learning. Hoboken: Taylor \& Francis.

Fischer, H.E., Klemm, K., Leutner, D., Sumfleth, E., Tiemann, R., \& Wirth, J. (2003). Naturwissenschaftsdidaktische Lehr-Lernforschung. Defizite und Desiderata. Zeitschrift für Didaktik der Naturwissenschaften, 9, 179-209.

Fischler, H. (2000a). Über den Einfluß von Unterrichtserfahrungen auf die Vorstellungen vom Lehren und Lernen bei Lehrerstudenten der Physik. Teil 1: Stand der Forschung sowie Ziele und Methoden einer Untersuchung. Zeitschrift für Didaktik der Naturwissenschaften, 6, 27-36.

Fischler, H. (2000b). Über den Einfluß von Unterrichtserfahrungen auf die Vorstellungen vom Lehren und Lernen bei Lehrerstudenten der Physik. Teil 2: Ergebnisse der Untersuchung. Zeitschrift für Didaktik der Naturwissenschaften, 6, 79-95.

Flick, U. (2014). Gütekriterien qualitativer Sozialforschung. In N. Baur \& J. Blasius (Hrsg.), Handbuch Methoden der empirischen Sozialforschung (S. 411-423). Wiesbaden: Springer Fachmedien $\mathrm{GmbH}$.

Gaudin, C., \& Chaliès, S. (2015). Video viewing in teacher education and professional development. A literature review. Educational Research Review, 16, 41-67. https://doi.org/10.1016/j.edurev. 2015.06.001.

Gerstenmaier, J., \& Mandl, H. (1995). Wissenserwerb unter konstruktivistischer Perspektive. Zeitschrift für Pädagogik, 41(6), 867-888.

Gomez-Zwiep, S. (2008). Elementary teachers' understanding of students' science misconceptions: implications for practice and teacher education. Journal of Science Teacher Education, 19(5), 437-454. https://doi.org/10.1007/s10972-008-9102-y.

Gropengießer, H. (1997). Schülervorstellungen zum Sehen. Zeitschrift für Didaktik der Naturwissenschaften, 3(1), 71-87.

Gropengießer, H. (1999). Was die Sprache über unsere Vorstellungen sagt. Kognitionslinguistische Analyse als Methode zur Erfassung 
von Vorstellungen: Das Beispiel Sehen. Zeitschrift für Didaktik der Naturwissenschaften, 5(2), 57-77.

Gropengießer, H. (2008). Qualitative Inhaltsanalyse in der fachdidaktischen Lehr-Lernforschung. In P. Mayring \& M. Gläser-Zikuda (Hrsg.), Die Praxis der Qualitativen Inhaltsanalyse (S. 172-189). Weinheim: Beltz.

Gropengießer, H., \& Marohn, A. (2018). Schülervorstellungen und Conceptual Change. In D. Krüger, I. Parchmann \& H. Schecker (Hrsg.), Theorien in der naturwissenschaftsdidaktischen Forschung (S. 49-67). Berlin, Heidelberg: Springer Berlin Heidelberg.

Grüß-Niehaus, T., \& Schanze, S. (2011). Eine kategoriegestützte Übersicht von Lernervorstellungen zum Löslichkeitsbegriff. CHEMKON, 18(1), 19-26. https://doi.org/10.1002/ckon.201010 141.

Gurel, D. K., Eryilmaz, A., \& McDermott, L.C. (2015). A review and comparison of diagnostic instruments to identify students' misconceptions in science. EURASIA Journal of Mathematics, Science \& Technology Education. https://doi.org/10.12973/ eurasia.2015.1369a.

Hammann, M., \& Asshoff, R. (2017). Schülervorstellungen im Biologieunterricht. Ursachen für Lernschwierigkeiten (3. Aufl.). Seelze: Klett.

Hascher, T. (2008). Diagnostische Kompetenzen von Lehrpersonen. In C. Kraler \& M. Schratz (Hrsg.), Wissen erwerben, Kompetenzen entwickeln. Modelle zur kompetenzorientieren Lehrerbildung (S. 71-86). Münster: Waxmann.

Hashweh, M.Z. (1996). Effects of science teachers' epistemological beliefs in teaching. Journal of Research in Science Teaching, 33(1), 47-63. https://doi.org/10.1002/(SICI)1098-2736(199601) 33:1〈47::AID-TEA3〉3.0.CO;2-P.

Heeg, J., Bittorf, R.M., \& Schanze, S. (2020a). Lernendenvorstellungen zum chemischen Gleichgewicht - ein systematisches Review. CHEMKON, 27(8), 373-383. https://doi.org/10.1002/ ckon.201900022.

Heeg, J., Hundertmark, S., \& Schanze, S. (2020b). The interplay between individual reflection and collaborative learning-seven essential features for designing fruitful classroom practices that develop students' individual conceptions. Chemical Education in Research and Practice, 21(3), 765-788. https://doi.org/10.1039/ C9RP00175A.

Heeg, J., Bittorf, R. M., \& Schanze, S. (im Druck). Förderung der Diagnosefähigkeiten angehender Lehrkräfte hinsichtlich Lernendenvorstellungen. Anwendung der Didaktischen Rekonstruktion zur Gestaltung eines hochschuldidaktischen Seminares. Herausforderung Lehrer_innenbildung.

Heinrichs, H. (2015). Diagnostische Kompetenz von Mathematik-Lehramtsstudierenden. Messung und Förderung (Perspektiven der Mathematikdidaktik). Dissertation. Wiesbaden: Springer Spektrum.

Heitzmann, N., Seidel, T., Hetmanek, A., Wecker, C., Fischer, M.R., Ufer, S., Schmidmaier, R., Neuhaus, B., Siebeck, M., Stürmer, K., Obersteiner, A., Reiss, K., Girwidz, R., Fischer, F., \& Opitz, A. (2019). Facilitating diagnostic competences in simulations in higher education a framework and a research agenda. Frontline Learning Research. https://doi.org/10.14786/flr.v7i4.384.

Helmke, A. (2009). Unterrichtsqualität und Lehrerprofessionalität (2. Aufl.). Diagnose, Evaluation und Verbesserung des Unterrichts.

Herppich, S., Praetorius, A.-K., Hetmanek, A., Glogger-Frey, I., Ufer, S., Leutner, D., Behrmann, L., Böhmer, I., Böhmer, M., Förster, N., Kaiser, J., Karing, C., Karst, K., Klug, J., Ohle, A., \& Südkamp, A. (2017). Ein Arbeitsmodell für die empirische Erforschung der diagnostischen Kompetenz von Lehrkräften. In A. Südkamp \& A.-K. Praetorius (Hrsg.), Diagnostische Kompetenz von Lehrkräften. Theoretische und methodische Weiterentwicklungen (S. 75-93). Münster: Waxmann.
Hofman, R., \& Roth, J. (2018). Assessment of students' thinking when working with graphs of functions-Promoting pre-service teachers' diagnostic competence. CERME 2017, Dublin, Ireland.

Hoppe, T., Renkl, A., \& Rieß, W. (2020). Förderung von unterrichtsbegleitendem Diagnostizieren von Schülervorstellungen durch Video- und Textvignetten. Unterrichtswissenschaft, 48, 573-597.

Horstkemper, M. (2006). Fördern heißt diagnostizieren. Pädagogische Diagnostik als wichtige Voraussetzung für individuellen Lernerfolg. Friedrich Jahresheft, 24, 4-7.

Hößle, C., Hußmann, S., Michaelis, J., Niesel, V., \& Nührenbörger, M. (2017). Fachdidaktische Perspektive auf die Entwicklung von Schlüsselkenntnissen einer förderorientierten Diagnostik. In C. Selter, S. Hußmann, C. Hößle, C. Knipping, K. Lengnink \& J. Michaelis (Hrsg.), Diagnose und Förderung heterogener Lerngruppen. Theorien, Konzepte und Beispiele aus der MINTLehrerbildung (S. 19-38). Münster: Waxmann.

Huang, R., Kulm, G., Li, Y., Smith, D., \& Bao, J. (2011). Impact of video case studies on elementary mathematics teachers' ways of evaluating lessons. An exploratory study. The Mathematics Educator, 13(1), 53-71.

Hübner, S., Nückles, M., \& Renkl, A. (2007). Lerntagebücher als Medium des selbstgesteuerten Lernens. Wie viel instruktionale Unterstützung ist sinnvoll? Empirische Pädagogik, 21(2), 119-137.

Ilyas, A., \& Saeed, M. (2018). Exploring teachers' understanding about misconceptions of secondary grade chemistry students. International Journal for Cross-Disciplinary Subjects in Education, 9(1), 3323-3328.

Ingenkamp, K., \& Lissmann, U. (2008). Lehrbuch der pädagogischen Diagnostik (6. Aufl.). Weinheim: Beltz Verl.

Janik, T., Minariková, E., \& Najvar, P. (2013). Der Einsatz von Videotechnik in der Lehrerbildung. Eine Übersicht leitender Ansätze. In U. Riegel \& K. Macha (Hrsg.), Videobasierte Kompetenzforschung in den Fachdidaktiken. Fachdidaktische Forschungen, (Bd. 4, S. 63-78). Münster: Waxmann Verlag GmbH.

Jeffries, C., \& Maeder, D. W. (2005). Using vignettes to build and assess teacher understanding of instructional strategies. Professional Educator, 27, 17-28.

Jelemenska, P. (2012). Lehrervorstellungen zum Lehren und Lernen von Evolution. Eine Fallstudie zum fachdidaktischen Coaching. Zeitschrift für Didaktik der Naturwissenschaften, 18, 229-259.

Johannsmeyer, F., Schneider, J., \& Oetken, M. (2003). Schülervorstellungen zum Boyle-Versuch. CHEMKON, 10(2), 73-74. https:// doi.org/10.1002/ckon.200390023.

Johnson, R. B., \& Onwuegbuzie, A. J. (2004). Mixed methods research: a research paradigm whose time has come. Educational Researcher, 33(7), 14-26. https://doi.org/10.3102/0013189X033007014.

Johnson, H. J., \& Cotterman, M.E. (2015). Developing preservice teachers' knowledge of science teaching through video clubs. Journal of Science Teacher Education, 26(4), 393-417. https://doi.org/ 10.1007/s10972-015-9429-0.

Kagan, D. M. (1992). Professional growth among preservice and beginning teachers. Review of Educational Research, 62(2), 129-169. https://doi.org/10.3102/00346543062002129.

Karst, K. (2012). Kompetenzmodellierung des diagnostischen Urteils von Grundschullehrern. Empirische Erziehungswissenschaft, Bd. 35. Münster: Waxmann.

Karst, K., Schoreit, E., \& Lipowsky, F. (2014). Diagnostische Kompetenzen von Mathematiklehrern und ihr Vorhersagewert für die Lernentwicklung von Grundschulkindern. Zeitschrift für pädagogische Psychologie, 28(4), 237-248. https://doi.org/10.1024/ 1010-0652/a000133.

Kattmann, U., Duit, R., Gropengießer, H., \& Komorek, M. (1997). Das Modell der Didaktischen Rekonstruktion. Ein Rahmen für naturwissenschaftsdidaktische Forschung und Entwicklung. Zeitschrift für Didaktik der Naturwissenschaften, 3(3), 3-18.

Kember, D. (1997). A reconceptualisation of the research into university academics' conceptions of teaching. Learning and Instruction, 7(3), 255-275. https://doi.org/10.1016/S0959-4752(96)00028-X. 
Kerr, K., Beggs, J., \& Murphy, C. (2006). Comparing children's and student teachers' ideas about science concepts. Irish Educational Studies, 25(3), 289-302. https://doi.org/10.1080/03323310600 913732.

Kleickmann, T., Vehmeyer, J. K., \& Möller, K. (2010). Zusammenhänge zwischen Lehrervorstellungen und kognitivem Strukturieren im Unterricht am Beispiel von Scaffolding-Maßnahmen. Unterrichtswissenschaft, 38(3), 210-228.

Kleinknecht, M., \& Gröschner, A. (2016). Fostering preservice teachers' noticing with structured video feedback: results of an online- and video-based intervention study. Teaching and Teacher Education, 59, 45-56. https://doi.org/10.1016/j.tate.2016.05.020.

Klinghammer, J., Rabe, T., \& Krey, O. (2016). Unterrichtsbezogene Vorstellungen von Lehramtsstudierenden der Physik. Zeitschrift für Didaktik der Naturwissenschaften, 22(1), 181-195. https:// doi.org/10.1007/s40573-016-0049-9.

Koballa, T., Graber, W., Coleman, D. C., \& Kemp, A.C. (2000). Prospective gymnasium teachers' conceptions of chemistry learning and teaching. International Journal of Science Education, 22(2), 209-224. https://doi.org/10.1080/095006900289967.

Kopp, I., Encke, A., \& Lorenz, W. (2002). Leitlinien als Instrument der Qualitätssicherung in der Medizin Das Leitlinienprogramm der Arbeitsgemeinschaft Wissenschaftlicher Medizinischer Fachgesellschaften (AWMF): Das Leitlinienprogramm der Arbeitsgemeinschaft Wissenschaftlicher Medizinischer Fachgesellschaften (AWMF). Bundesgesundheitsblatt, Gesundheitsforschung, Gesundheitsschutz, 45(3), 223-233. https://doi.org/10.1007/s00103002-0378-3.

Krammer, K., \& Reusser, K. (2005). Unterrichtsvideos als Medium der Aus- und Weiterbildung von Lehrpersonen. Beiträge zur Lehrerbildung, 23(1), 35-50.

Krammer, K., Ratzka, N., Klieme, E., Lipowsky, F., Pauli, C., \& Reusser, K. (2006). Learning with classroom videos: conception and first results of an online teacher-training program. ZDM, 38(5), 422-432. https://doi.org/10.1007/BF02652803.

Kuckartz, U. (2018). Qualitative Inhaltsanalyse. Methoden, Praxis, Computerunterstützung (4. Aufl.). Weinheim: Beltz Juventa.

Kultusministerkonferenz (2017). Ländergemeinsame inhaltliche Anforderungen für die Fachwissenschaften und Fachdidaktiken in der Lehrerbildung. Berlin, Bonn: KMK.

Kunter, M., \& Trautwein, U. (2013). Psychologie des Unterrichts. Paderborn: Schöningh (UTB).

Larkin, D. (2012). Misconceptions about "misconceptions". Preservice secondary science teachers' views on the value and role of student ideas. Science \& Education, 96(5), 927-959. https://doi.org/10. 1002/sce.21022.

Leavy, A. M., McSorley, F. A., \& Boté, L. A. (2007). An examination of what metaphor construction reveals about the evolution of preservice teachers' beliefs about teaching and learning. Teaching and Teacher Education, 23(7), 1217-1233. https://doi.org/10.1016/j. tate.2006.07.016.

Lelgemann, M., \& Ollenschläger, G. (2006). Evidenzbasierte Leitlinien und Behandlungspfade: Ergänzung oder Widerspruch? Der Internist, 47(7), 690, 692-690, 697. https://doi.org/10.1007/ s00108-006-1652-5.

Levin, D. M., Hammer, D., \& Coffey, J.E. (2009). Novice teachers' attention to student thinking. Journal of Teacher Education, 60(2), 142-154. https://doi.org/10.1177/0022487108330245.

Lin, P.-J. (2005). Using research-based video-cases to help pre-service primary teachers conceptualize a contemporary view of mathematics teaching. International Journal of Science and Mathematics Education, 3(3), 351-377. https://doi.org/10.1007/s10763004-8369-5.

Lin, J.-W., \& Chiu, M.-H. (2007). Exploring the characteristics and diverse sources of students' mental models of acids and bases. International Journal of Science Education, 29(6), 771-803. https:// doi.org/10.1080/09500690600855559.
Llinares, S., \& Valls, J. (2009). The building of pre-service primary teachers' knowledge of mathematics teaching: interaction and online video case studies. Instructional Science, 37(3), 247-271. https:// doi.org/10.1007/s11251-007-9043-4.

Löfström, E., \& Poom-Valickis, K. (2013). Beliefs about teaching: persistent or malleable? A longitudinal study of prospective student teachers' beliefs. Teaching and Teacher Education, 35, 104-113. https://doi.org/10.1016/j.tate.2013.06.004.

Lohmann, G. (2006). Didaktische Rekonstruktion in der Hochschuldidaktik. Journal für Lehrerinnen- und Lehrerbildung, 2, 65-73.

Loibl, K., Leuders, T., \& Dörfler, T. (2020). A framework for explaining teachers' diagnostic judgements by cognitive modeling (DiacoM). Teaching and Teacher Education, 91, 103059.

Lucero, M. M., Petrosino, A. J., \& Delgado, C. (2017). Exploring the relationship between secondary science teachers' subject matter knowledge and knowledge of student conceptions while teaching evolution by natural selection. Journal of Research in Science Teaching, 54(2), 219-246. https://doi.org/10.1002/tea.21344.

Markic, S., \& Eilks, I. (2008). Unterrichtsbezogene Vorstellungen von Lehramtsstudierenden der Chemie am Beginn ihres Studiums und ihre Einordnung. CHEMKON, 15(2), 69-74. https://doi.org/10. 1002/ckon.200810072.

Markic, S., \& Eilks, I. (2013). Potential changes in prospective chemistry teachers' beliefs about teaching and learning-A crosslevel study. International Journal of Science and Mathematics Education, 11(4), 979-998. https://doi.org/10.1007/s10763-0139417-9.

Marohn, A. (2008). „Choice2learn“ - eine Konzeption zur Exploration und Veränderung von Lernervorstellungen im naturwissenschaftlichen Unterricht. Zeitschrift für Didaktik der Naturwissenschaften, 14, 57-83.

Marohn, A., \& Rohrbach, F. (2013). Professionalisierung in der Lehrerausbildung. Entwicklung und Evaluation eines am Forschenden Lernen orientierten Seminars zum Thema Schülervorstellungen im Chemieunterricht. Chimica et ceterae artes rerum naturae didacticae, 38(105), 21-46.

Mayring, P. (2015). Qualitative Inhaltsanalyse. Grundlagen und Techniken. Beltz Pädagogik, Bd. 12. Weinheim: Beltz.

McElvany, N., Schroeder, S., Hachfeld, A., Baumert, J., Richter, T., Schnotz, W., Horz, H., \& Ullrich, M. (2009). Diagnostische Fähigkeiten von Lehrkräften. Zeitschrift für pädagogische Psychologie, 23(34), 223-235. https://doi.org/10.1024/1010-0652.23.34. 223.

Meister, S., Nitz, S., Schwanewedel, J., \& Upmeier zu Belzen, A. (2020). Diagnostische Fähigkeiten Lehramtsstudierender - Förderung mit Videovignetten und Anwendung im Lehr-Lern-Labor. In B. Priemer \& J. Roth (Hrsg.), Lehr-Lern-Labore (Bd. 16, S. 223-247). Berlin, Heidelberg: Springer.

Mellado, V. (1997). Preservice teachers' classroom practice and their conceptions of the nature of science. Science \& Education, 6(4), 331-354.

Mellado, V., Bermejo, M.L., Blanco, L.J., \& Ruiz, C. (2007). The classroom practice of a prospective secondary biology teacher and his conceptions of the nature of science and of teaching and learning science. International Journal of Science and Mathematics Education, 6(1), 37-62. https://doi.org/10.1007/s10763-0079081-z.

Merseth, K. K. (1994). Cases, case methods, and the professional development of educators. ERIC digest

Meyer, H. (2004). Novice and expert teachers' conceptions of learners' prior knowledge. Science Education, 88(6), 970-983.

Meyer, H., Tabachnick, B.R., Hewson, P.W., Lemberger, J., \& Park, H.-J. (1999). Relationships between prospective elementary teachers' classroom practice and their conceptions of biology and of teaching science. Science Education, 83(3), 323-346.

Morrison, J. A., \& Lederman, N. G. (2003). Science teachers' diagnosis and understanding of students' preconceptions. Science Education, 87(6), 849-867. https://doi.org/10.1002/sce.10092. 
Naah, B. M. (2015). Enhancing Preservice teachers' understanding of students' misconceptions in learning chemistry. Journal of College Science Teaching, 45(2), 41-47.

Niebert, K., \& Gropengießer, H. (2014). Leitfadengestützte Interviews. In D. Krüger, I. Parchmann \& H. Schecker (Hrsg.), Methoden in der naturwissenschaftsdidaktischen Forschung (S. 121-132). Berlin Heidelberg: Springer Spektrum. https://doi.org/10.1007/ 978-3-642-37827-0_10.

Niebert, K., \& Gropengießer, H. (2015). Understanding starts in the mesocosm. Conceptual metaphor as a framework for external representations in science teaching. International Journal of Science Education, 37(5-6), 903-933. https://doi.org/10.1080/ 09500693.2015.1025310.

Niebert, K., Marsch, S., \& Treagust, D. F. (2012). Understanding needs embodiment. A theory-guided reanalysis of the role of metaphors and analogies in understanding science. Science \& Education, 96(5), 849-877. https://doi.org/10.1002/sce.21026.

Norton, L., Richardson, T.E., Hartley, J., Newstead, S., \& Mayes, J. (2005). Teachers' beliefs and intentions concerning teaching in higher education. Higher Education, 50(4), 537-571. https://doi. org/10.1007/s10734-004-6363-z.

Nussbaum, J. (1981). Towards the diagnosis by science teachers of pupils' misconceptions. An exercise with student teachers. European Journal of Science Education, 3(2), 159-169. https://doi. org/10.1080/0140528810030206.

Olson, J. K., Bruxvoort, C.N., \& Haar, V. A. J. (2016). The impact of video case content on preservice elementary teachers' decisionmaking and conceptions of effective science teaching. Journal of Research in Science Teaching, 53(10), 1500-1523. https://doi.org/ 10.1002/tea.21335.

Onwu, G. O., \& Randall, E. (2006). Some aspects of students' understanding of a representational model of the particulate nature of matter in chemistry in three different countries. Chemistry Education Research and Practice, 7(4), 226-239. https://doi.org/10. 1039/B6RP90012G.

Oser, F. (1997). Standards in der Lehrerbildung. Teil 1: Berufliche Kompetenzen, die hohen Qualitätsmerkmalen entsprechen. Beiträge zur Lehrerinnen- und Lehrerbildung, 15(1), 26-37.

Oser, F., Salzmann, P., \& Heinzer, S. (2009). Measuring the competence-quality of vocational teachers. An advocatory approach, $1(1), 65-83$.

Otero, V. K., \& Nathan, M. J. (2008). Preservice elementary teachers' views of their students' prior knowledge of science. Journal of Research in Science Teaching, 45(4), 497-523. https://doi.org/10. 1002/tea.20229.

Özdemir, G., \& Clark, D. B. (2007). An overview of conceptual change theories. EURASIA Journal of Mathematics, Science \& Technology Education, 3(4), 351-361.

Özmen, H. (2004). Some student misconceptions in chemistry: a literature review of chemical bonding. Journal of Science Education and Technology, 13(2), 147-159.

Pajares, M.F. (1992). Teachers' beliefs and educational research: cleaning up a messy construct. Review of Educational Research, 62(3), 307-332. https://doi.org/10.3102/00346543062003307.

Park, S., \& Oliver, J. S. (2008). Revisiting the conceptualisation of pedagogical content knowledge (PCK): PCK as a conceptual tool to understand teachers as professionals. Research in Science Education, 38(3), 261-284.

Patry, J.-L. (2016). Thesen zur konstruktivistischen Didaktik. Journal für Lehrerinnen- und Lehrerbildung, 16(2), 9-17.

Perleth, M., Jakubowski, E., \& Busse, R. (2000). „Best Practice“ im Gesundheitswesen - oder warum wir evidenzbasierte Medizin, Leitlinien und Health Technology Assessment brauchen. Zeitschrift für Ärztliche Fortbildung und Qualitätssicherung, 94(9), 741-744.

Petermann, K., Friedrich, J., \& Oetken, M. (2010). Diagnose-Test zur Erhebung von Schülervorstellungen zum Themenfeld ,Massenerhalt bei chemischen Reaktionen“. PdN-ChiS, 4(59), 34-37.
Pietzner, V. (2015). Das Wissen von Chemielehrkräften über Schülervorstellungen. In K. Höner, M. Looß, R. Müller \& A. Strahl (Hrsg.), Naturwissenschaften vermitteln. Von der frühen Kindheit bis zum Lehrerberuf. Naturwissenschaften vermitteln Brauschweiger Beiträge zu Lehrerbildung und Fachdidaktik, (Bd. 5, S. 176-206).

Pilitsis, V., \& Duncan, R. G. (2012). Changes in belief orientations of preservice teachers and their relation to inquiry activities. Journal of Science Teacher Education, 23(8), 909-936. https://doi.org/10. 1007/s10972-012-9303-2.

Pine, K., Messer, D., \& John, S. K. (2001). Children's misconceptions in primary science: a survey of teachers' views. Research in Science \& Technological Education, 19(1), 79-96. https://doi.org/ 10.1080/02635140120046240.

Postareff, L., Katajavuori, N., Lindblom-Ylänne, S., \& Trigwell, K. (2008a). Consonance and dissonance in descriptions of teaching of university teachers. Studies in Higher Education, 33(1), 49-61. https://doi.org/10.1080/03075070701794809.

Postareff, L., Lindblom-Ylänne, S., \& Nevgi, A. (2008b). A followup study of the effect of pedagogical training on teaching in higher education. Higher Education, 56(1), 29-43. https://doi.org/10. 1007/s10734-007-9087-z.

Praetorius, A.-K., \& Südkamp, A. (2017). Eine Einführung in das Thema der diagnostischen Kompetenz von Lehrkräften. In A. Südkamp \& A.-K. Praetorius (Hrsg.), Diagnostische Kompetenz von Lehrkräften. Theoretische und methodische Weiterentwicklungen (S. 13-18). Münster: Waxmann.

Praetorius, A.-K., Lipowsky, F., \& Karst, K. (2012). Diagnostische Kompetenz von Lehrkräften: Aktueller Forschungsstand, unterrichtspraktische Umsetzbarkeit und Bedeutung für den Unterricht. In Differenzierung im mathematisch-naturwissenschaftlichen Unterricht (S. 115-146).

Putnam, R. T., \& Borko, H. (1997). Teacher learning: implications of new views of cognition. In B. J. Biddle, T. L. Good \& I. F. Goodson (Hrsg.), International handbook of teachers and teaching (S. 1223-1296). Dordrecht: Springer Netherlands.

Rath, V. (2017). Diagnostische Kompetenz von angehenden Physiklehrkräften. Dissertation. Berlin: Logos.

Reiners, C. S. (2017). Chemie vermitteln. Berlin, Heidelberg: Springer.

Reinfried, S., Aeschbacher, U., Kienzler, P.M., \& Tempelmann, S. (2013). Mit einer didaktisch rekonstruierten Lernumgebung Lernerfolge erzielen. das Beispiel Wasserquellen und Gebirgshydrologie. Zeitschrift für Didaktik der Naturwissenschaften, 19, 259-286.

Reinfried, S., Mathis, C., \& Kattmann, U. (2009). Das Modell der Didaktischen Rekonstruktion - eine innovative Methode zur fachdidaktischen Erforschung und Entwicklung von Unterricht. Beiträge zur Lehrerbildung, 27(3), 404-414.

Richardson, V., \& Kile, R.S. (1999). Learning from videocases. In M. A. Lundeberg (Hrsg.), Who learns what from cases and how? The research base for teaching and learning with cases (S. 121-136). Mahwah: Erlbaum.

Riemeier, T. (2005). Schülervorstellungen von Zellen, Teilung und Wachstum. Zeitschrift für Didaktik der Naturwissenschaften, 11, 41-55.

Riemeier, T. (2007). Moderater Konstruktivismus. In D. Krüger \& H. Vogt (Hrsg.), Theorien in der biologiedidaktischen Forschung. Ein Handbuch für Lehramtsstudenten und Doktoranden (S. 69-80). Berlin, Heidelberg: Springer.

Riemeier, T. (2010). Wo das Blut fließt. Schülervorstellungen zu Blut, Herz und Kreislauf beim Menschen. Zeitschrift für Didaktik der Naturwissenschaften, 16, 77-92.

Rieu, A., Loibl, K., Leuders, T., \& Herppich, S. (2020). Diagnostisches Urteilen als informationsverarbeitender Prozess - Wie nutzen Lehrkräfte ihr Wissen bei der Identifizierung und Gewichtung von Anforderungen in Aufgaben? Unterrichtswissenschaft. https://doi.org/10.1007/s42010-020-00071-x. 
Sadler, P. M., Sonnert, G., Coyle, H. P., Cook-Smith, N., \& Miller, J. L. (2013). The influence of teachers' knowledge on student learning in middle school physical science classrooms. American Educational Research Journal, 50(5), 1020-1049. https://doi.org/10. 3102/0002831213477680.

Santagata, R., \& Angelici, G. (2010). Studying the impact of the lesson analysis framework on preservice teachers' abilities to reflect on videos of classroom teaching. Journal of Teacher Education, 61(4), 339-349. https://doi.org/10.1177/0022487110369555.

Schaefer, S., Blomberg, G., Stürmer, K., \& Seidel, T. (2012). Der Einsatz von Lerntagebüchern in der universitären Lehrerausbildung. Welchen Effekt haben strukturierende Leitfragen auf die Reflexionsfähigkeit von Lehramtsstudierenden? Empirische Pädagogik, 26(2), 271-291.

Schäfer, S., \& Seidel, T. (2015). Noticing and reasoning of teaching and learning components by pre-service teachers. Journal for educational research online, 7(2), 34-58.

Schlichter, N. (2012). Lehrerüberzeugungen zum Lehren und Lernen. Dissertation. Göttingen: Georg-August-Universität Göttingen.

Schmelzing, S., van Driel, J.H., Jüttner, M., Brandenbusch, S., Sandmann, A., \& Neuhaus, B.J. (2013). Develeopment, evaluation, and validation of a paper-and-pencil test for measuring two components of biology teachers' pedagogical content knowledge concerning the "cardiovascular system". International Journal of Science and Mathematics Education, 11(6), 1369-1390.

Schrader, F.-W. (2013). Diagnostische Kompetenz von Lehrpersonen. Beiträge zur Lehrerinnen- und Lehrerbildung, 31(2), 154-165.

Schrader, F.-W. (2014). Lehrer als Diagnostiker. In E. Terhart, H. Bennewitz \& M. Rothland (Hrsg.), Handbuch der Forschung zum Lehrerberuf (S. 683-698). Münster: Waxmann.

Seago, N. (2004). Using video as an object of inquiry for mathematics teaching and learning. In J. Brophy (Hrsg.), Using video in teacher education (S. 259-286). Oxford: Elsevier.

Seidel, T., Schwindt, K., Rimmele, R., \& Prenzel, M. (2009). Konstruktivistische Überzeugungen von Lehrpersonen: Was bedeuten sie für den Unterricht? In M. A. Meyer, M. Prenzel \& S. Hellekamps (Hrsg.), Perspektiven der Didaktik (S. 259-276). Wiesbaden: VS.

Seifried, J., \& Wuttke, E. (2017). Der Einsatz von Videovignetten in der wirtschaftspädagogischen Forschung: Messung und Förderung von fachwissenschaftlichen und fachdidaktischen Kompetenzen angehender Lehrpersonen. In C. Gräsel \& K. Trempler (Hrsg.), Entwicklung von Professionalität pädagogischen Personals (S. 303-322). Wiesbaden: Springer.

Sherin, M. G., Linsenmeier, K. A., \& van Es, E. A. (2009). Selecting video clips to promote mathematics teachers' discussion of student thinking. Journal of Teacher Education, 60(3), 213-230. https:// doi.org/10.1177/0022487109336967.

Sherin, M. G., \& van Es, E. A. (2005). Using video to support teachers' ability to notice classroom interactions. Journal of Technology and Teacher Education, 13(3), 475-491.

Shulman, L.S. (1987). Knowledge and teaching: foundations of the new reform. Harvard Educational Review, 57(1), 1-22.

Siemes, A. (2008). Diagnosetheorien. In S. Kliemann (Hrsg.), Diagnostizieren und Fördern in der Sekundarstufe I. Schülerkompetenzen erkennen, unterstützen und ausbauen (S. 12-21). Berlin: Cornelsen Scriptor.

Smith III, J. P., diSessa, A. A., \& Roschelle, J. (1993). Misconceptions reconceived: a constructivist analysis of knowledge in transition. Journal of the Learning Sciences, 3(2), 115-163. https://doi.org/ $10.1207 / \mathrm{s} 15327809 \mathrm{jl} 1 \mathrm{~s} 0302$ _ 1.

Star, J.R., \& Strickland, S. K. (2008). Learning to observe: using video to improve preservice mathematics teachers' ability to notice. Journal of Mathematics Teacher Education, 11(2), 107-125. https://doi.org/10.1007/s10857-007-9063-7.

Stark, R. (2003). Conceptual Change. Kognitiv oder situiert? Zeitschrift für pädagogische Psychologie, 17(2), 133-144. https:// doi.org/10.1024//1010-0652.17.2.133.
Taber, K. S. (1997). Student understanding of ionic bonding: molecular versus electrostatic thinking? School Science Review, 78(285), $85-95$.

Taber, K. S. (2013). Revisiting the chemistry triplet. Drawing upon the nature of chemical knowledge and the psychology of learning to inform chemistry education. Chemistry Education Research and Practice, 14(2), 156-168. https://doi.org/10.1039/C3RP00012E.

Taber, K. S. (2019). Alternative conceptions and the learning of chemistry. Israel Journal of Chemistry, 59(6-7), 450-469. https://doi. org/10.1002/ijch.201800046.

Uhren, S., Ralle, B., \& Di Fuccia, D. (2013). Zum Umgang von Lehrkräften mit alternativen Schülervorstellungen. chimica et ceterae artes rerum naturae didacticae, 38, 5-20.

Upmeier zu Belzen, A., \& Merkel, R. (2014). Einsatz von Fällen in der Lehr- und Lernforschung. In D. Krüger, I. Parchmann \& H. Schecker (Hrsg.), Methoden in der naturwissenschaftsdidaktischen Forschung (S. 203-212). Berlin, Heidelberg: Springer Spektrum.

Vosniadou, S. (2013). Conceptual change in learning and instruction. The framework theory approach. In S. Vosniadou (Hrsg.), International handbook of research on conceptual change (S. 11-30). Hoboken: Taylor \& Francis.

Voss, T., Kleickmann, T., Kunter, M., \& Hachfeld, A. (2013). Mathematics teachers' beliefs. In M. Kunter, J. Baumert, W. Blum, U. Klusmann, S. Krauss \& M. Neubrand (Hrsg.), Cognitive activation in the mathematics classroom and professional competence of teachers (S. 249-271). New York: Springer. https://doi.org/10. 1007/978-1-4614-5149-5_12.

Wandersee, J., Mintzes, J. J., \& Novak, J. D. (1994). Research on alternative conceptions in science. In D. L. Gabel (Hrsg.), Handbook of research on science teaching and learning. A project of the National Science Teachers Association (S. 177-210). New York: Macmillan.

Wedel, A., Müller, C.R., Pfetsch, J., \& Ittel, A. (2019). Training teachers' diagnostic competence with problem-based learning: a pilot and replication study. Teaching and Teacher Education, 86, 102909. https://doi.org/10.1016/j.tate.2019.102909.

Weinert, F. E., Schrader, F.-W., \& Helmke, A. (1990). Educational expertise. Closing the gap between educational research and classroom practice. School Psychology International, 11(3), 163-180. https://doi.org/10.1177/0143034390113002.

Widodo, A., \& Duit, R. (2004). Konstruktivistische Sichtweisen vom Lehren und Lernen und die Praxis des Physikunterrichts. Zeitschrift für Didaktik der Naturwissenschaften, 10, 233-255.

Widodo, A., \& Duit, R. (2005). Konstruktivistische Lehr-Lern-Sequenzen und die Praxis des Physikunterrichts. Zeitschrift für Didaktik der Naturwissenschaften, 11, 131-146.

Wildgans-Lang, A., Scheuerer, S., Obersteiner, A., Fischer, F., \& Reiss, K. (2020). Analyzing prospective mathematics teachers' diagnostic processes in a simulated environment. ZDM. https://doi.org/ 10.1007/s11858-020-01139-9.

Wong, L. S., Yung, W. B. H., Cheng, M.W., Lam, K. L., \& Hodson, D. (2006). Setting the stage for developing pre-service teachers' conceptions of good science teaching: the role of classroom videos. International Journal of Science Education, 28(1), 1-24. https:// doi.org/10.1080/09500690500239805.

Yang, C., Noh, T., Scharmann, L.C., \& Kang, S. (2014). A study on the elementary school teachers' awareness of students' alternative conceptions about change of states and dissolution. The AsiaPacific Education Researcher, 23(3), 683-698. https://doi.org/10. 1007/s40299-013-0140-7.

Zhang, M., Lundeberg, M., Koehler, M. J., \& Eberhardt, J. (2011). Understanding affordances and challenges of three types of video for teacher professional development. Teaching and Teacher Education, 27(2), 454-462. https://doi.org/10.1016/j.tate.2010.09.015. 